\title{
Italique
}

Poésie italienne de la Renaissance

XV | 2012

Varia

\section{Il sonetto LXII di Giovanni della Casa e l'epilogo del suo «Canzoniere»}

\section{Francesco Bausi}

\section{OpenEdition}

\section{Journals}

\section{Edizione digitale}

URL: http://journals.openedition.org/italique/343

DOI: $10.4000 /$ italique.343

ISSN: 1663-4438

\section{Editore}

Librairie Droz

\section{Edizione cartacea}

Data di pubblicazione: 1 dicembre 2012

Paginazione: 11-46

ISBN: 978-2-600-01639-1

ISSN: 1423-3983

Notizia bibliografica digitale

Francesco Bausi, « II sonetto LXII di Giovanni della Casa e l'epilogo del suo «Canzoniere» », Italique [Online], XV | 2012, online dal 01 décembre 2015, consultato il 02 mai 2019. URL : http:// journals.openedition.org/italique/343 ; DOI : 10.4000/italique.343 


$$
\text { FRA NCESCO B A US I }
$$

\section{IL SONETTO LXII DI GIOVANNI \\ DELLA CASA E L'EPILOGO \\ DEL S U O «CANZON IERE»}





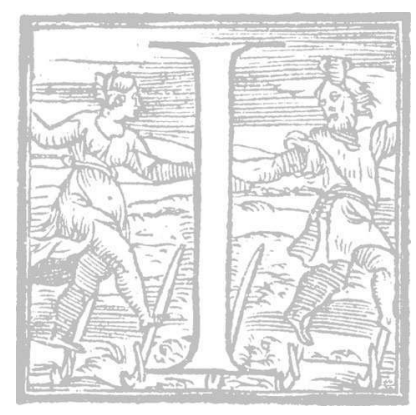

l sonetto LXII, terżultimo delle rime casiane secondo la stampa veneziana del I5) 8 curata due anni dopo la morte del poeta dal suo segretario Erasmo Gemini, può considerarsi una sorta di summa dell'intero canzoniere, di cui compendia al tempo stesso le principali tematiche e le più peculiari soluzioni formali. Esso può fornire dunque una privilegiata porta d'accesso alla complessa arte di Giovanni Della Casa, e insieme consentire - per la sua particolare posizione in seno al libro - di mettere in luce le raffinate dinamiche strutturali della sua sezione conclusiva. ${ }^{\mathrm{I}}$

Già lessi, ed or conosco in me, sì come Glauco nel mar si pose uom puro e chiaro, e come sue sembianze si mischiaro di spume e conche, e fêrsi alga sue chiome; però che 'n questo Egeo che vita ha nome puro anch'io scesi e 'n queste de l'amaro mondo tempeste, ed elle mi gravaro i sensi e l'alma, ahi, di che indegne some!

Lasso! e soviemmi d'Esaco, che l'ali d'amoroso pallor segnate ancora digiuno per lo cielo apre e distende, e poi, satollo, indarno a volar prende: sì 'l core anch'io, che per sé leve fora, gravato ho di terrene esche mortali.

Il componimento è occupato dall'evocazione di due miti classici, simmetricamente distribuiti nelle sue due parti: nelle quartine, il mito del pescatore Glauco, che avendo visto $i$ pesci da lui catturati tornare miracolosamente in vita, volle mangiare l'erba sulla quale li aveva deposti, e si trasformò in un dio marino; nelle terzine, il mito di Esaco, il quale, in preda alla disperazione per aver indirettamente causato la morte dell'amata Esperia (uccisa da un serpente mentre egli la inseguiva), decise di gettarsi in mare, ma venne salvato da Tetide, che, impietositasi, lo trasformò in un uccello marino, lo smergo. Miti narrati, com'è noto, da Ovidio nelle Metamorfosi, rispettivamente nel libro XIII (vv. 898-968) e nel libro XI (vv. 749-95). In entrambi $i$ casi, la 
rapida allusione mitologica è seguita dalla sua interpretazione allegorica in senso morale e autobiografico: ${ }^{2}$ nel primo caso, il poeta si paragona a Glauco perché come quest'ultimo, diventando abitatore dei mari, si ricopri di conchiglie, sassi e alghe, cosi egli, facendosi travolgere dalle tempeste della vita, ha gravato il corpo e l'anima di impurità e peccati; nel secondo caso, l'analogia è indicata nel fatto che Esaco, divenuto uccello, può volare solo se digiuno, mentre non riesce a farlo quando è sazio, allo stesso modo del poeta, che ha appesantito il suo cuore, di per sé puro e leggero, con gli appetiti materiali, e dunque non è in grado di staccarsi dai beni terreni. ${ }^{3}$

La sommaria esposizione appena tentata tradisce e banalizza, però, la straordinaria ricchezza semantica e l'elaboratissima tessitura formale del componimento. Ho parlato di Ovidio: va detto subito, però, che come da più parti è stato osservato - tanto nell'esposizione, quanto nell'interpretazione dei due miti, il Casa si discosta dalla sua fonte primaria. Nelle Metamorfosi, infatti, Glauco viene accolto fra le divinità marine, e la sua è presentata come una divinizzazione, analogamente a quanto fa Dante nel primo del Paradiso (dove quello di Glauco è l'exemplum di cui il poeta si serve per rendere l'idea del «trasumanare», ossia dell'altrimenti indescrivibile passaggio dell'uomo alla condizione divina): ${ }^{4}$

Beatrice tutta ne l'etterne rote fissa con li occhi stava; e io in lei le luci fissi, di là sù rimote.

Nel suo aspetto tal dentro mi fei, qual si fé Glauco nel gustar de l'erba che 'l fé consorto in mar de li altri dèi.

Trasumanar significar per verba non si poria; però l'essemplo basti a cui esperienza grazia serba.

(Dante, Paradiso I 64-72)

Qui, invece, l'esperienza del mitico pescatore è interpretata in modo opposto, come un imbestiamento, un allontanamento dalla perfezione originaria, una perdita dell'humanitas e dunque della stessa divinitas, essendo l'uomo fatto a immagine e somiglianza di Dio. ${ }^{6}$ Quanto ad Esaco, Ovidio non dice che egli, divenuto smergo, voli soltanto se digiuno, mentre non possa farlo se sazio, ma si limita ad affermare che 
per desiderio di morte - essendo ancora innamorato di Esperia continua a ripetere il suo gesto e a tuffarsi sotto le onde del mare, perché quello è l'elemento che egli ama. Un poeta umanista quale è il Casa, però, può discostarsi dalle sue fonti solo se autorizzato da altre non meno autorevoli fonti. Riguardo a Glauco, egli si appoggia - come già videro $i$ commentatori cinque e seicenteschi - a un passo della Repubblica platonica, dove Glauco rappresenta l'anima dell'uomo che ba perduto la sua costitutiva purezza a causa degli elementi esterni che l'hanno corrotta e sfigurata in seguito alla sua unione con il corpo e ai vizi da tale unione scaturiti:

Il ragionamento appena concluso e altri del genere possono dunque indurre ad affermare l'immortalità dell'anima. $\mathrm{Ma}$, al contrario di quanto facciamo noi, non si deve osservare la sua vera natura ora che è guastata dall'unione con il corpo e con gli altri vizi; occorre osservarla allo stato puro, con gli occhi dell'intelletto. Allora si troverà che essa è molto più bella, e distingueremo con maggiore chiarezza la giustizia, l'ingiustizia e tutto ciò che abbiamo già detto. Le nostre parole sono vere in rapporto al suo stato presente; e in effetti l'abbiamo vista nella condizione di Glauco marino. Difficilmente si potrebbe vedere ancora la sua natura originaria, perché delle vecchie parti del suo corpo alcune sono state spaccate, altre schiacciate e completamente sfigurate dalle onde. Ma si sono aggiunti nuovi elementi: conchiglie, alghe, sassi, sicché Glauco assomiglia più a un altro essere qualsiasi che a quello che era originariamente. Allo stesso modo, anche noi vediamo l'anima ora che è oppressa da infiniti mali. Ma bisogna guardare, Glaucone, in un'altra direzione. [...] Verso il suo amore per la sapienza. E occorre comprendere ciò che essa comprende e quali compagnie desidera, dato che è affine a ciò che è divino, immortale ed eterno, e quale potrebbe essere se seguisse tale principio, condotta da tale slancio fuori dal mare in cui ora si trova, e si scuotesse d'intorno i sassi e le conchiglie che ora la ricoprono, portati dal fango di cui si nutre, da quei materiali terrosi e rocciosi, molteplici e selvaggi, che provengono dai cosiddetti festini beati. Allora si potrebbe scorgere come sia la sua vera natura, complessa oppure semplice, e di quali elementi si componga.

(Platone, Repubblica x, 6i I b-e $)^{7}$

Per Esaco, $i$ commentatori moderni non rinviano ad alcuna fonte, ma in verità essa era stata precocemente individuata da Pompeo Garigliano, autore nel I6Is di una lettura di questo sonetto edita con altre 
sue l'anno successivo a Napoli (e importante per il rinvio a numerose fonti greche e per l'attenta lettura filosofica e specificamente platonica del componimento): ${ }^{8}$ si tratta delle Enneadi di Plotino (V, 9, I), «il quale - scrive Garigliano - distinguendo nel libro dell'intelletto e delle idee gli uomini in contemplativi, attivi e voluttuosi, asserisce questi ultimi assomigliarsi a quelli uccelli di rapina, $i$ quali quanto più sono carichi di preda, tanto meno possono volare».? Secondo Plotino, in effetti, gli nomini immersi nella realtà sensibile e dediti solo ai piaceri sono come uccelli appesantiti dalla preda, cosicché, pur dotati di ali, non riescono a volare, cioè a innalżarsi al di sopra della terra per tornare a Dio attraverso la vita contemplativa:

Tutti gli uomini dalla nascita usano i sensi prima dell'intelligenza e incontrano innanzitutto necessariamente gli oggetti sensibili; gli uni restano fermi ad essi passandovi tutta la vita, ritenendo che siano le prime e le ultime cose e sostenendo che il dolore e il piacere che si trovano in esse siano il male e il bene; pensano che questo basti e trascorrono la vita bramando l'uno e allontanando l'altro. Tra di essi poi chi si appropria del ragionare afferma che questa sia la sapienza, come uccelli pesanti che hanno tratto molto dalla terra $\mathrm{e}$, trovandosi così appesantiti, non sono in grado di volare in alto, benché abbiano ricevuto le ali dalla natura. Altri si alzano un poco dal basso, perché la parte più elevata della loro anima li spinge dal piacere alla bellezza; tuttavia, dato che non sono capaci di guardare in alto e non hanno altro cui appoggiarsi, precipitano, insieme con il nome della virtù, verso la prassi, verso la scelta tra le cose di quaggiù, da cui prima avevano tentato di innalzarsi. Vi è poi una terza specie di uomini divini, di maggior forza e dallo sguardo più acuto, che contemplano, come per una grande acutezza visiva, il fulgore di lassù e s'innalzano quasi sopra le nubi e l'oscurità terrena e permangono là, disprezzando tutte le cose del mondo e rallegrandosi di quel luogo vero e familiare, come un uomo che, dopo molto vagare, sia ritornato alla sua patria, governata da buone leggi. ${ }^{\circ}$

E Plotino, si badi, recupera da Platone anche la lettura in chiave "degradante" del mito di Glauco, che parimenti egli intende (Enneadi I, I, I2) come metafora della sourapposizione, all'anima umana non composta, di scorie e aggiunte ad essa estranee, da cui l'uomo deve purificarsi, se vuole riguadagnare la semplicità e la perfezione originaria. ${ }^{\text {II }}$ Le implicazioni platonizzanti di questo sonetto 
furono còlte già dai primi lettori ed esegeti; ${ }^{\mathrm{I} 2}$ né la forte caratura filosofica del componimento può stupire, in un poeta, come il Casa, la cui biblioteca era particolarmente ricca sul versante della filosofia antica, e soprattutto di quella platonica e neoplatonica. ${ }^{13}$

Specialmente indicativa, sotto questo profilo, è la prima stesura del dialogo tassiano Il Gonzaga o vero del piacer onesto (I580), dove l'aristotelico Agostino Nifo, accingendosi a una sottilissima illustrazione filosofica del sonetto LXII, definisce l'autore «nobilissimo poeta ed oratore, e de gli occulti misteri non meno de la filosofia che de la poetica conoscitore». ${ }^{{ }^{4} 4}$ Qui, verso la fine, $i$ due interlocutori (il Nifo, chiamato Agostino Sessa dal nome della sua città natale, e Cesare Gonzaga) si intrattengono a lungo sull'esegesi allegorico-filosofica del nostro sonetto e in particolare della figura mitologica di Glauco ( $v v$. I-8, riferiti dal Gonzaga), interpretandola nello stesso senso del Casa, quello platonico. A parere del Nifo, Glauco è l'uomo che gusta il piacere sensuale (figurato dall'erba) e salta nel mare (cioè si abbandona ai piaceri carnali, figurati dal mare, dalla cui spuma nacque Venere) tanto da diventare quasi bruto; 's o per meglio dire è l'anima - ossia l'intelletto puro ed immortale - che scende nel corpo, perché il corpo è materia dell'anima, e la materia è dai filosofi tradizionalmente assimilata all'acqua, per cui il Casa paragona la vita del corpo al mare (Petrarca aveva usato l'immagine analoga del torrente). ${ }^{16}$ I vv. 3-4 del sonetto descrivono appunto l'intelletto che, scendendo nel corpo, si mescola con l'anima sensitiva e con quella vegetativa, rappresentate da schiume, conchiglie e alghe: le conchiglie sono animali, e designano pertanto l'anima sensitiva; le alghe sono vegetali, e stanno per l'anima vegetativa; la spuma è la schiuma donde, secondo il mito, nacque Venere (lo sperma, cioè), e simboleggia dunque il piacere dei sensi.

Il Gonzaga, citando Paradiso I 67-72, obietta che Glauco, per Dante, non è l'uomo vinto dal piacere sensuale al punto da trasformarsi in bestia, ma, viceversa, l'uomo che si trasmuta in Dio. Nifo replica che in effetti il mito di Glauco può interpretarsi anche in altro modo: Glauco è l'intelletto che discende nel corpo; il mare ove egli pesca è il soggetto della filosofia naturale, il suo pescare è il sillogizzare dell'intelletto, la rete è la logica, i pesci sono gli universali e le conclusioni vere cui egli giunge, l'erba è il piacere della contemplazione, grazie alla quale egli si deifica, perché la felicità contemplativa rende l'uomo divino. Questa seconda interpretazione (quella dantesca) sarà poi preferita da Tasso, 
che commentando i vv. I2-I4 del proprio sonetto Erba felice, che già in sorte avesti ${ }^{17}$ ("Già, novo Glauco, in ampio mar mi spazio / d'immensa gioia, e 'n più tranquillo stato / quasi mi par ch'immortal forma i' prenda») afferma di adottare l'interpretazione dantesca del mito (che allude alla deificazione di Glauco, come anche in Ovidio), non quella platonica, seguita invece dal Casa. E ciò spiega perché, nelle successive redazioni del dialogo, Tasso abbia espunto l'esegesi platonizzante del casiano sonetto LXII.

L'originalità del Casa, come di norma, è dunque un'originalità essenzialmente "combinatoria", che consiste nella disposizione inedita di componenti topiche. Essa risiede da un lato nell' aver connesso il mito di Esaco con l'immagine plotiniana degli uccelli appesantiti dal cibo, paragonati dal filosofo agli nomini materiali (il trait d'union gli venne fornito probabilmente da Plinio il Vecchio, Nat. hist. XI, 202, che colloca lo smergo, insieme alla lince, fra gli animali più voraci e insaziabili, perché in loro il cibo passa direttamente dallo stomaco nell'intestino); ${ }^{18}$ dall'altro, nell'aver accostato $i$ miti di Glauco ed Esaco, grazie a un'interpretazione allegorica che ne evidenzia, ai suoi occhi, le affinità profonde. Si tratta infatti di due miti che per il Casa alludono al motivo platonico della caduta dell'anima, all'idea dell'uomo, cioè, che perde la sua primitiva perfezione e piomba nell'opacità della materia; ${ }^{19}$ due miti, potremmo dire, della "pesantezza" che aggrava, e che toglie all'anima quelle ali di cui parla appunto Platone nel Fedro e che le sono necessarie per risalire verso Dio. ${ }^{20}$ Il nesso fra $i$ due miti, insomma, è costituito dal fatto che Glauco si copre di scorie che lo sfigurano, ed Esaco si appesantisce col cibo: in entrambi i casi, si tratta di elementi esterni e impuri che compromettono la perfezione originaria dell'anima. ${ }^{21}$

Ma i due miti presentano anche altre analogie che certamente attirarono l'attenzione del poeta, e lo spinsero ad avvicinarli in questo sonetto. Entrambi, infatti, sono miti del mare e della gola. Il mare è quello in cui Glauco ed Esaco si immergono, con un movimento discendente del corpo che si contrappone all'ascesa dell'anima a Dio (e il Casa colse certamente il contatto testuale per antitesi ravisabile a questo proposito in Ovidio, per il quale Glauco «corpusque sub aequora mersit», mentre di Esaco dice che «corpus super aequora mittit»); quel mare in cui vivono tanto i pesci divorati dallingordo Esaco, quanto le conche e le alghe che aggravano e deturpano Glauco (simbolo, gli uni e le altre, dei 
"pesanti" beni terreni); quel mare che lo stesso poeta qui vede, del tutto tradizionalmente, come allegoria della vita terrena, delle sue tempeste e delle passioni da essa scatenate nel cuore dell'nomo ${ }^{22}$ (v. 5: «questo Egeo che vita ha nome», dove Egeo è normale sineddoche classicheggiante per 'mare', come Euro per 'vento' nel sonetto successivo, v. I3: "ché più crudo Euro a me mio verno adduce»). ${ }^{23}$ La gola, invece, è il peccato che accomuna le due figure: di Esaco, come abbiamo visto, il Casa sottolinea - sulla scorta di Plinio - la voracità, tale da impedirgli, quando è satollo, di spiccare il volo, al pari degli uccelli di rapina di cui parla Plotino; di Glauco, è ben noto (tanto che il poeta trascura di accennarvi) che la sua metamorfosi fu causata dal desiderio di mangiare l'erba miracolosa dai quali i pesci morti avevano ottenuto nuovamente la vita. Cosicché Glauco poteva apparire al Casa una sorta di Adamo punito per il suo "ardito gusto» (Par. XXXII I22), parimenti dovuto alla superbia di voler conseguire l'immortalità e diventare un dio ("eritis sicur diì, dice il serpente a Eva nella Genesi 3, 5). D'altronde, la tematica del nostro sonetto è non meno cristiana che platonica: basti rinviare a Sapienza, 9, Is ("Corpus enim quod corrumpitur aggravat animam, et terrena inhabitatio deprimit sensum multa cogitantem») ${ }^{24} e$ al De consolatione boeziano (V, m. 5, I3-Is: "qui recto caelum vultu petis exserisque frontem, / in sublime feras animum quoque, ne gravata pessum / inferior sidat mens corpore celsius levato»), passi donde il Casa recupera il verbo gravare che ricorre ben due volte nel sonetto, ai vv. 7 e 14.

Del resto, nei versi casiani fortissima è l'insistenza sulla colpa e sulla responsabilità dell'uomo: Glauco, scrive infatti il poeta, si pose in mare, con un'espressione affine a quella ovidiana («corpusque sub aequora mersi»), ma che - nella sintetica narrazione del sonetto LXII, dove ̀̀ omessa la descrizione della metamorfosi - sottolinea marcatamente l'elemento volontaristico della scelta individuale da parte dell'uomo che decide di rinunciare alla sua primigenia purezza per lasciarsi contaminare dalle «indegne some» mortali. Altri elementi comuni ai due miti sono poi l'acqua e l'erba, parimenti portatrici di topiche valenze simboliche. Glauco ed Esaco si innamorano entrambi di una fanciulla che scorgono lungo l'acqua del mare o di un fiume: quell'acqua che, come sottolinea il Nifo nel dialogo tassiano, rappresenta la "materia". Quanto all'erba, il serpente che uccide Esperia sta nascosto nell'erba, come la virtù miracolosa che fa rivivere i pesci ma che causerà l'imbes- 
tiamento di Glauco: e l'erba è radice di morte, giacché, come sappiamo, è fra l'erba, ossia fra le belle ma ingannevoli apparenze dei beni terreni, che si nasconde il serpente-diavolo (si pensi al mito di Orfeo ed Eudirice - con quest'ultima che, al pari di Esperia, viene morsa e uccisa dal serpente mentre fugge il suo amante - e alle sue interpretazioni allegoriche). ${ }^{25}$

Le rime del Casa, come il loro supremo modello, i petrarcheschi Fragmenta, ricorrono con molta parsimonia alla mitologia. Se si escludono sparsi e isolati accenni, solo in un altro testo, il sonetto XXXVI (La bella greca), la componente mitologica appare davvero dominante e decisiva, giacché il poeta paragona lì la dedicataria Elisabetta Quirini a tre mitiche bellezze dell'antichità (Elena, Semele e Dafne) che a suo parere, se si sottoponessero al giudizio di Paride, sarebbero - come ogni altra bella donna - da lei superate. Ma nel sonetto XXXVI $i$ paragoni mitologici sono di tipo cortigiano e di fattura del tutto tradizionale, tanto che l'intero componimento si configura come la ripresa e la variazione di un tema topico, già recuperato - per ricordare solo due testi tenuti ben presenti dal Casa - in un'ottava del Furioso $e$ in un sonetto di Pietro Bembo (il I33). ${ }^{26}$ Il sonetto LXII, invece, rivela l'inclinazione a rivivere il mito in una prospettiva drammaticamente soggettiva $e$ autobiografico-morale, sulla scorta della canzone XXIII dei Fragmenta, che ne costituisce il modello diretto: potremmo dire, anzi, che il nostro sonetto è l'equivalente casiano della canzone delle metamorfosi, quasi una sua moderna riprodurione in scala ridotta. ${ }^{27}$

In quella canzone, infatti, Petrarca rappresenta cinque momenti della sua dolorosa vicenda amorosa attraverso altrettante metamorfosi mitologiche desunte da Ovidio, dichiarando di aver vissuto di persona le sconvolgenti esperienze di Dafne, Cicno, Batto, Biblide e Atteone. In entrambi i poeti, il mito metamorfico diviene formalizzazione e correlativo oggettivo di esperienze vissute, ${ }^{28}$ offrendosi come exemplum $e$ al tempo stesso come unico strumento attraverso il quale il poeta può rappresentare situazioni psicologiche "estreme" e pertanto diversamente inesprimibili (al pari del Dante "trasumanato" che si paragona a Glauco), grazie a un "immaginario" e a un linguaggio largamente condivisi e dunque immediatamente comprensibili al lettore. Inoltre, canzone e sonetto insistono con dolente meraviglia sul passaggio dalla lettura alla vera conoscenza: il primo verso del sonetto LXII ("Già lessi, ed or conosco in me») si impernia sulla distinzione fra un passato in cui 
$i$ testi letterari erano stati fruiti in modo epidermico (per mero diletto o pura erudizione, evidentemente) e un presente in cui l'autore invece finalmente comprende, sperimentandolo sulla propria pelle, il senso profondo di ciò che in precedenza aveva si letto, ma senza trarne profitto e (come avrebbe detto Machiavelli) farne capitale. Analogamente Petrarca, nella canzone XXIII, dopo aver rievocato la propria trasformazione in fonte (sulla scorta del mito di Biblide), constata stupito come quella storia, che fino ad allora gli era parsa un'inverosimile favola, possieda un contenuto di verità prima insospettato, ma di cui egli ha poi personalmente fatto diretta esperienza: "Chi udi mai d'uom vero nascer fonte? / E parlo cose manifeste et contes) ${ }^{29}$ ( $v v$. IIg-20, dove la $\mathrm{E}$ che inaugura il v. I20 vale 'eppure', e sottolinea, col suo forte valore avversativo, il contrasto fra l'apparente inverosimiglianza della favola e la cruda realtà del proprio vissuto)..$^{30}$

Il sonetto casiano, sotto questo profilo, è poi certo memore anche di un'altra lirica dei Fragmenta, il sonetto LVI, che si conclude con questi versi:

Et or di quel ch'i' ò lecto mi sovene, che 'nanzi al dì de l'ultima partita huom beato chiamar non si convene.

Versi donde proviene al Casa del sonetto LXII non solo la mossa del v. I «ed on», ma anche il soviemmi del v. g, e nei quali l'allusione è a un notissimo detto di Solone già ripreso in forma sintetica nella stessa canzone XXIII (v. 3I: "La vita el fin, e'l di loda la sera») e tramandato da innumerevoli fonti greche e latine, fra cui spiccano ancora una volta le ovidiane Metamorfosi, cui verosimilmente il Petrarca si riferisce quando dice «io lecto» (Metam. III 135-37: «sed scilicet ultima semper / exspectanda dies homini est, dicique beatus / ante obitum nemo supremaque funera debet»). Ma petrarchesco, benché di derivazione senechiana e agostiniana, è anche il motivo della necessità di passare dalla scienza alla sapienza, dall'erudizione all'etica, di non limitarsi ciò̀ a studiare e a leggere, ma di preoccuparsi piuttosto di ricavare dalle proprie letture insegnamenti utili al perfezionamento morale: un tema, questo, che percorre tutto il Secretum, dove Agostino più volte rimprovera Francesco di non saper tradurre le sua vasta cultura letteraria in una salutare regola di vita, e dichiara che a niente giova aver tanto 
letto, se non sappiamo metterlo in pratica. Cosi nel terzo libro del dialogo, dove Agostino cita due versi ovidiani (Rem. am. 579-580; "Quisquis amas, loca sola nocent, loca sola caveto. I Quo fugis? In populo tutior esse potes») e, dopo che Francesco ha affermato di conoscerli perfettamente sin dallinfanzia ("Recordor optime: ab infantia pene michi familiariter noti erant»), esclama: "Quid multa nosse profuit, si ea necessitatibus tuis accomodare nescivisti?»). ${ }^{\text {I }}$ Sempre nel terzo libro del dialogo si manifesta con chiarezza a Francesco la differenza e la distanza cronologica tra $i$ due momenti: quello, lontano, della lettura senza profitto, e quello, presente, dell'esperienza personale, che gli consente di comprendere il senso profondo dei testi. Si veda quanto Petrarca scrive a proposito della lettera pitagorica:

Litere velut Pithagoree, quam audivi et legi, non inanem esse doctrinam. Cum enim recto tramite ascendens ad bivium pervenissem modestus et sobrius, et dextram iuberer arripere, ad levam - incautus dicam an contumax? - deflexi; neque michi profuit quod sepe puer legeram:

Hic locus est partes ubi se via findit in ambas;

dextera que Ditis magni sub menia ducit.

Hac iter Elysium nobis; at leva malorum

exercet penas, et ad impia Tartara mittit. (Aen. vI 540-43)

Hec nimirum, quanquam ante legissem, non tamen prius intellexi quam expertus sum. Ex tunc autem obliquo sordidoque calle distractus et sepe retro lacrimans conversus, dextrum iter tenere non potui, quod cum deserui, tunc, profecto tunc, fuerat illa morum meorum facta confusio. ${ }^{32}$

Lo spunto torna moltissime altre volte, sia nel secondo libro ${ }^{33}$ che ancora nel terzo, dove, accanto ad altri passi, ${ }^{34}$ conviene sottolineare almeno il seguente:

Aug. Quo enim spectat labor iste perpetuus continuequeque vigilie ac vehemens impetus studiorum? Respondebis forsitan, ut vite tue profutura condiscas. At vero iam pridem vite simul et morti necessaria didicisti. Erat igitur potius quemadmodum in actum illa produceres experiendo tentandum, quam in laboriosa cognitione procedendum, ubi novi semper recessus et inaccesse latebre et inquisitionum nullus est terminus. $^{35}$

Ma si potrebbe citare anche il De remediis, in cui si afferma esplicitamente che la «notitia litterarum» è davvero proficua solo quando 
trascorre a norma dell'azione. Cosi a I 44, 5-6 ("G. Scribo libros. R. Melius fortassis illos legeres, optime autem in vite regulam lecta converteres: tunc enim est utilis notitia litterarum, dum in actum transit»), ${ }^{36}$ e soprattutto a II 9, I3-I4:

Dolor. Rerum atque animi inops sum.

Ratio. Primum te expeditum, secundum plane inopem facit et miserum. Sed ut Aristippi consilium, sic et Theophrasti dictum te legisse arbitror. Quid vero lectio sola profuerit? Et meminisse oportet, et in usus tuos lecta convertere. ${ }^{37}$

E, questo, uno spunto quanto mai attinente allo svolgimento del canzoniere del Casa, la cui parte finale trova uno dei suoi temi portanti nel superamento dell'aspirazione alla gloria poetica: un superamento che non comporta per lui, ovviamente, la rinuncia alla letteratura, quanto piuttosto il rifiuto di una concezione puramente "esteriore" di essa (intesa, come nel petrarchesco Secretum, quale strumento privilegiato per soddisfare la curiositas e conseguire la fama), ora abbandonata a favore di un approccio capace di attingere la "sostanza" eticoesistenziale dei testi, ${ }^{3}$ sgombrando il campo da "accidenti", orpelli ed elementi superflui (quasi che anche la poesia, al pari della vita, fosse un Glauco cui è necessario sottrarre scorie e impurità, con un michelangiolesco levare richiamato opportunamente da Giuliano Tanturli per il sonetto LXIV, ultimo della silloge). ${ }^{39}$

Per tutto questo, il sonetto LXII di Giovanni Della Casa si rivela intimamente "petrarchesco", ben al di là, intendo dire, dell'adozione di un "linguaggio" (lessico, immagini, movenze ritmico-sintattiche) modellato in gran parte su quello dei Fragmenta, e del fatto che nel capitolo II del Triumphus Cupidinis si trovino a breve distanza, privi tuttavia di qualunque interpretazione allegorica, $i$ miti di Esaco e di Glauco (vv. I60-62 e I72-74). ${ }^{40}$ Ciò che distingue il Monsignore dal suo auctor di riferimento è però - e non solo in questo componimento - l'evidente tendenza a drammatizzare $i$ contenuti etico-filosofici del testo poetico esasperando l'enfasi del discorso. Innanzitutto, emerge chiaramente qui come in tutto il canzoniere del Casa - la tendenza alla potente concentrazione formale ed espressiva: ${ }^{4 I}$ l'ampia canzone XXIII dei Fragmenta diviene un sonetto, le cinque metamorfosi petrarchesche si riducono a due (la seconda delle quali, per giunta, solo rapidamente 
evocata), e il componimento ostenta perentorio, fin dal primo verso, l'interpretazione autobiografica ("Già lessi, ed or conosco in me») cui il poeta intende piegare i racconti mitologici di Glauco e di Esaco. Inoltre, si tratta - come già detto - di due metamorfosi affini, dal momento che esemplificano, in ordine inverso e dunque con effetto di ysteron proteron, le due tentazioni fondamentali (some o esche, come egli le definisce ai vv. 8 e I4) che hanno segnato la vita del Casa, l'amore e l'ambizione (rivolta, quest'ultima, a due oggetti distinti: gloria poetica e onori mondani): Glauco, infatti, si è trasformato in mostro marino per desiderio di immortalità (avendo voluto provare su di sé gli effetti dell'erba miracolosa che aveva ridato vita ai pesci morti), mentre Esaco è divenuto smergo in seguito al suo amore infelice ed eccessivo per Esperia. E si tratta, ancora una volta, delle due grandi tentazioni petrarchesche, le due «catene» del Secretum, un dialogo di cui, proprio in merito a questo tema, si sorprendono echi anche nella fondamentale canzone XLVII, ${ }^{42}$ che inaugura la svolta "penitenziale" e "morale" delle rime casiane. Fra $i$ due momenti, però, c'è anche una successione cronologica e logica: Glauco è l'uomo che si corrompe (rispetto alla sua purezza originaria) e resta nel mare, mentre Esaco è l'uomo che si pente e vorrebbe risalire (vale a dire, purificarsi), ma non riesce nel suo intento. Il sonetto, cosi, riproduce, nelle sue due fasi, il senso e la situazione di tutta la seconda parte delle rime (dalla canzone XLVII in poi), anzi il senso della stessa canzone XLVII: caduta, consapevolezza della colpa, ravvedimento, pentimento e aspirazione al cambiamento, conversione, mutatio vitae. ${ }^{43}$ Si noti, a questo riguardo, anche la differenza dei tempi: di Glauco si parla al passato (si pose, fêrsi), di Esaco al presente (apre e distende, prende). Come a dire che il Casa è stato Glauco - quando, sceso puro nel mondo, si è aggravato di pesi materiali - ed è ancora oggi Esaco, che riesce a volare solo se digiuno (giacché egli, non avendo in tutto compiuto il suo cammino di perfezionamento morale, conosce periodiche ricadute nel male).

Se dunque il primo è il mito della discesa inesorabile, il secondo è quello della risalita difficile (o impossibile), giusta il topos neoplatonico. In questo doppio movimento, Glauco scende nel mare (in basso), Esaco vorrebbe levarsi verso l'alto (in cielo), ma non può farlo perché ancora gravato dalle passioni, tanto che, secondo il mito, continua anche dopo la sua metamorfosi a portare $i$ segni della passione amorosa che lo ha reso macilento; allo stesso modo, il Casa dichiara, a XLVII 62, di essersi 
allontanato dall'amore «tardo [...] et lasso a lento volo», ma di fatto non afferma di aver vinto del tutto il desiderio amoroso (si veda inoltre XLVII 54-57; e anche nella canzone XXXII, pur proclamando che è ormai tempo per lui di liberarsi dai lacci d'amore, si definisce al v. 70 "canuto amante», e ai vv. 23-33 ammette di essere, benché vecchio, ancora preda dell'amore), ${ }^{44}$ cosi come, quando subentra il desiderio di onori (XLVII 86 ss.), non per questo egli dice di aver definitivamente messo da parte l'aspirazione alla fama poetica. Non si può escludere che qui l'amore di Esaco rappresenti in generale l'amore per $i$ beni terreni, e quindi, nello specifico, le ambizioni di carriera ecclesiastica, ultima "tentazione" del Casa; ma pare improbabile, perché il sonetto LXII è un sonetto riassuntivo, che fa il bilancio di una vita intera, e le some e le esche dei vv. 8 e it dovrebbero dunque alludere genericamente alle lusinghe terrene, di qualunque tipo, che aggravano l'anima (compresa la passione amorosa). Il Casa affermerebbe cioè che, come Esaco resta sempre segnato dall'amore, cosi egli continua ad essere macchiato dal desiderio di terrene esche mortali, fra cui - in questa estrema fase della sua vita - predominano il desiderio di gloria $e$ l'ambizione politica. ${ }^{45} \mathrm{Ma}$ l'ambiguità non sembra sanabile, e forse ̀̀ intenzionale.

Il sonetto LXII, insomma, si presenta come una sorta di testo-consuntivo, collocato com'è in una posizione strategica all'interno delle rime, dopo la sestina LXI (che proclama la rinuncia definitiva agli onori mondani) $e$ prima degli ultimi due sonetti, occupati nell'ordine dal pensiero della morte incombente e dalla rasserenante scoperta della presenza divina nel mondo $e$ in se stessi, ${ }^{46}$ col finale approdo alla vita contemplativa (nell'ultimo sonetto infatti il poeta, letteralmente, contempla l'universo e, cosi facendo, si innalza finalmente al di sopra della terra). Giova ancora rammentare, a questo proposito, i tre tipi umani di Plotino, che nell'interpretazione del Garigliano rappresentano i voluttuosi, gli attivi e $i$ contemplativi: proprio come nel canzoniere casiano, dominato dalle due tentazioni capitali (di cui la prima, l'amore, corrisponde al primo e più basso livello, quello della vita dei sensi, e la seconda, l'ambizione mondana, al secondo, quello della vita attiva), cui segue l'agognato traguardo della vita contemplativa (LXIII-LXIV). Questo, testimoniato dalla stampa del I558, è pertanto l'ordine corretto degli ultimi due sonetti - non quello, inverso, tràdito dal Chigiano O VI 80-perché, come in Petrarca, è solo dalla meditazione della morte incombente (LXIII) che l'uomo può trarre l'incentivo a staccarsi dai beni 
terreni, a convertirsi e a tornare a Dio (LXIV). ${ }^{47}$ Possiamo quindi sostenere che il sonetto LXII è il punto d'arrivo del percorso morale del libro, e pone a sua volta le premesse per la conclusione (LXIV), dopo la pausa del sonetto LXIII (presentimento dell'imminente morte). ${ }^{48}$

A conferma, si considerino $i$ nessi fra gli ultimi tre sonetti, nonché $i$ rapporti tra il LXII e le altre rime del libro. In primo luogo, va detto che $i$ due temi fondamentali del sonetto LXII caratterizzano l'intero canzoniere, soprattutto nella parte finale (dopo la svolta morale e penitenziale della canzone XLVII), e talora compaiono - come qui - nella medesima lirica:

I) la consapevolezza che la propria anima, pura in origine, si sia corrotta a causa delle impurità del mondo, e la conseguente aspirazione a mondarsi e a purgarsi, ricorrono con immagini simili a XVII I-4 («Io, che l'età solea viver nel fango, / Hoggi, mutato il cor da quel ch'i' soglio, / d'ogni immondo penser mi purgo et spoglio / e'l lungo mio fallir correggo et piango»); XXVI I-2 («Mentre fra valli paludose et ime / ritengon me larve turbate et mostri»); XLI 5-6 («O tempestosa, o torbida procella, / che 'n mar sì crudo la mia vita girilı); XLVII 4I-46 («[...] et ben convene / hor penitentia et duol l'anima lave / de' color atri et del terrestre limo, / ond'ella è per mia colpa infusa et grave, / ché se 'l ciel me la diè candida et leve, / terrena e fosca a lui salir non deve»), ${ }^{49}$ XLIX I-4, a Trifon Gabriele («Poco il mondo già mai t'infuse o tinse, / Triphon, ne l'atro suo limo terreno, / et poco inver' gli abissi onde egli è pieno / i puri et santi tuoi pensier' sospinse»); L 5-8 («[...] Iniqua parte / elegge ben chi il ciel chiaro e sovrano / lassa et gli abissi prende: ahi cieco humano / desir, che mal da terra si diparte»). Ma vd. anche XLVIII I-3 ("Come splende valor, perch'huom no 'l fasci / di gemme o d'ostro, et come ignuda piace / et negletta virtù pura et verace»), con la contrapposizione fra la virtù «pura e verace», che «ignuda e negletta» piace e risplende, e quella coperta invece di gemme e d'ostro (appesantita, offuscata, dunque, mentre Trifon Gabriele, ora che è morto, è scarco, alleggerito della soma terrena; idem a LXI 37-39: «Già in pretïoso cibo o 'n gonna d'oro / non crebbe, anzi tra querce e 'n povera esca, / virtù»). E naturalmente il sonetto LXIv, con la «pura parte di me» (l'anima) che la vita mortale, oscura e fredda, ha involto «ne l'atre nubi sue», e che ora il poeta riesce a liberare dall'antico fardello grazie alla contemplazione del creato, in cui Dio ha tratto la luce e l'aer puro (l'aggettivo torna ben due volte, ai vv. 3 e 9) da «abissi oscuri e misti», aprendo ciò che era chiuso nelle tenebre e 
che ora riluce in terra e in cielo: è la creazione del mondo dal caos, ed è ciò che l'uomo deve fare su se stesso, recuperando con l'aiuto divino, fra le tenebre del mondo e delle passioni, la sua purezza primigenia. ${ }^{50}$ L'uomo che esce dalla nubi della vita materiale e mortale (vv. I-4) è come il cielo e la luce che Dio ha estratto dalle tenebre primordiali: nella corrispondenza perfetta fra macrocosmo e microcosmo, la storia del singolo riflette e riproduce, ogni volta, la storia del creato, e ciò le conferisce senso e la avvalora, perché l'uomo comprende che anche i suoi peccati fanno parte del disegno divino di salvezza. Come dal caos, infatti, Dio ha saputo "estrarre" la bellezza e l'ordine del mondo, così dai peccati del passato l'uomo può risalire verso il bene.

2) il tema dell'uccello appesantito e dunque impossibilitato a volare (come lo smergo in cui si trasformò Esaco), ${ }^{\text {I }}$ cui il Casa paragona se stesso, desideroso ma incapace di sfuggire ai lacci terreni, domina nella parte finale delle rime. ${ }^{52}$ Cfr. XIX 9-I I; XXXII 59-63; XLVII 3 I-32 («corsi, com'augel sòle / che d'alto scenda et a suo cibo vole») e soprattutto 6I-66 («con la dolce esca ond'ei [scil. Amore] pascendo strugge, / tardo partimmi et lasso a lento volo; / indi cantando il mio passato duolo, / in sé l'alma s'accolse / et di desir novo arse / credendo assai da terra alto levarse»); XuIX 5-I I, per la morte di Trifon Gabriele («Et hor di lui [scil. dal mondo] si scosse in tutto e scinse / tua candida alma, et leve fatta a pieno / salìo, son certo, ov'è più il ciel sereno, / et quanto lice più ver' Dio si strinse. / Ma io rassembro pur sublime augello / in ima valle preso, et queste piume / caduche homai pur anchor visco invoglia»); ${ }^{53} \mathrm{LI}$ Io-I4 ( $\mathrm{O}$ fosca, o senza luce / vista mortal, cui sì del mondo cale, / come non t'ergi al ciel, che sol produce / eterni frutti? Ahi vile augel su l'ale / pronto, ch'a terra pur si riconduce!»); LII 9-Iо («[...] Hor non s'arresta / spesso nel fango augel di bianche piume?»); LIII 5-6 («Ma io palustre augel, che poco s'erga / su l'ale, sembro», in contrapposizione al «nobil cigno» del v. I, che è Pietro Bembo); LXI I 3-Is («Io, come vile augel scende a poca esca / dal cielo in ima valle, i miei dolci anni / vissi in palustre limo», col ritorno del palustre limo e dell'immagine dell'uccello che per «poca esca» - cioè per l'effimera e falsa felicità promessa dai beni terreni - scende dal cielo in una valle oscura; il tema è una variante dell'altro, quello dell'uccello che rimane impigliato nel vischio e solo con gran fatica riesce a liberarsi). ${ }^{54} \mathrm{Si}$ noti infine che in precedenza il Casa aveva creduto di levarsi a volo grazie all'amore (XLIV I 2-I4) e soprattutto alla poesia (xxxv 8 e I 3 , dove il Bembo è detto «sacro cigno sublime»; XLVII 65-66: «et di desir novo arse [scil. l'anima del poeta] / credendo assai da terra alto levarse»), prima che anch'essa venisse res- 
pinta come un errore e un falso bene (vd. L I 2-I 4, con la netta antitesi fra la «celeste luce» e quella, debole, della poesia, che «poco a chiari farne [...] vale»). Per il motivo del "peso" vd. xxiII 9- 10 : il poeta, catturato nuovamente dall'amore, sottomette l'anima a un «novo incarco», e si trova così a dover sopportare una «doppia salma»; e anche il sonetto extravagante 78 , dove il poeta esorta il cardinale Ippolito d'Este a spronare i signori d'Italia affinché sgombrino le loro nobili anime «di sì gravi salme» (l'avarizia e la superbia dei vv. 5-6).

Il canzoniere del Casa, dominato da una serratissima intratestualità, ̀̀ un universo ristretto e fortemente concentrato, ove ricorrono in modo ossessivo pochi temi e costanti parole-chiave, immagini e metafore. ${ }^{5}$ Qui, in particolare, tutto si fonda sulla figura dell'antitesi: puroimpuro, leggero-pesante, alto-basso, digiuno-sazio, leggere-conoscere, chiaro-scuro (ossia, luce-ombra: un contrasto, questo, sviluppato più a fondo nel sonetto LXIV, che su di esso è interamente imperniato; e si ricordi anche la canzone XLVII, dove il poeta afferma ai vv. 93-94 che l'ambizione trasforma i giorni sereni in notti scure e dolorose, e prima, $v v$. 56-57, che l'amore volge in tenebre ciò che dà luce all'anima, ossia la ragione). Inoltre, il tema della metamorfosi lega i sonetti LXII-LXIII, ${ }^{56}$ posto che nel LXIII il poeta si sente trasformare in ghiaccio (IO-II: "ret ghiaccio / gli spirti anch'io sento et le membra farsi»), ${ }^{57}$ quel ghiaccio della morte che parimenti getta un ponte verso il sonetto LXIV (dove quella viva morte che è la vita mortale «'n una o 'n due / brevi e notturne hore trapassa», e viene definita «oscura / et fredda»: vv. I-3). Né si dimentichi la canzone XLVI, che fa posto ( $v v$. 60-74) alla rievocazione di tre celebri metamorfosi, quelle di Dafne, Anassarete e Narciso: personaggi mitologici puniti per aver rifiutato l'amore, e la cui sorte il poeta auspica anche per la donna da lui amata. ${ }^{58}$

Molto stretti, come stiamo vedendo, sono in particolare $i$ nodi che legano il sonetto LXII al LXIV. Abbiamo già avuto modo di constatare come il motivo dell'esca e del cibo inscriva il sonetto LXII nell'area tematica della gola e dunque dell'incontinenza: Esaco «satollo» è infatti figura dell'uomo travolto e "aggravato" dalle passioni e dagli appetiti, e la sua "dismisura" ci respinge per contrasto al sonetto LXIV, al centro del quale $(v .7)$ si staglia viceversa la «dolce del ciel legge e misura», che governa il mondo e che alla fine il poeta scopre essere «magisterio» di Dio, anzi Dio stesso (Sapienza II, 2I: «omnia in mensura, et numero 
et pondere disposuisti»). Inoltre, lo spunto iniziale ("Già lessi, ed or conosco») del nostro sonetto tornerà identico nella fronte del LXIV, imperniata sulla medesima contrapposizione tra passato e presente: "Questa vita mortal [...] involto bavea fin qui la pura / parte di me nell'atre nubi sue. // Or a mirar le grazie tante tue / prendo» (vv. I-б). E quel che giustamente dice Tanturli a proposito della «straordinaria forza liberatoria» dell'attacco del $v$. 5 del sonetto conclusivo può bene trasferirsi anche all'incipit del LXII, dove parimenti si dà conto di un'autentica "conversione", di una vera e propria svolta esistenziale. ${ }^{59}$

Si ponga mente, poi, ai nessi testuali e metaforici che collegano il sonetto LXII ad altri componimenti delle rime, soprattutto ai testi immediatamente adiacenti:

- I Già: riprende il Già di LXI 37 (attacco del congedo della sestina: «Già in pretioso cibo o 'n gonna d'oro»).

- 2 puro e chiaro: vd. XXIV I-4: il poeta visse lieto e pieno di speranza i pochi giorni puri e sereni che il cielo assegnò alla sua vita oscura: «Nessun lieto già mai né 'n sua ventura / pago né pien, com'io, di speme visse / i pochi dì ch'a la mia vita oscura / puri et sereni il ciel parco prescrisse». Inoltre, nel sonetto LXIV l'anima è definita «la pura / parte di me» (vv. 3-4), e chiara è la luce creata da Dio (v. Io). ${ }^{60}$

- 3 si mischiaro: vd. LXIV I I: «abissi oscuri e misti» (con l'immagine del trarre la luce dagli abissi, e dunque l'idea della risalita dal mare alla superficie, del passaggio dall'informe alla forma, dall'impurità alla purezza, dalle tenebre alla luce: anche qui la creazione come un trarre, uno sprigionare la forma, cioè l'anima, dal peso opaco della materia). D'altronde, nel sonetto conclusivo «la pura parte» dell'uomo involta nelle nubi della vita mortale corrisponde a Glauco coperto dalle impurità del mare; e gli abissi oscuri e misti donde Dio ha tratto il cielo e la luce corrispondono al mare in cui si sprofondano Glauco ed Esaco: come Dio ha tratto da quegli abissi la luce, così l'uomo, al pari di Esaco, deve risalire da quel mare verso il cielo.

- s Egeo: per un'analoga sineddoche vd. Euro (per 'venti' in generale) a LXIII I 3. L'intero v. 5, come notano i commentatori, è esemplato su Petrarca, Tr. Etern. 47-48: «di questo alpestro e rapido torrente / ch'à nome vita» (Della Casa sostituisce il torrente col mare, come nota GARIGLIANO, p. 49). ${ }^{6 \mathrm{I}}$

- 6-7 le tempeste del mondo: vd. Xvi 6-8: «Ma non commosser mai contrari venti / onda di mar, come le nostre menti / con le tempeste sue 
conturba Amore» (così dice il cuore, dialogando con il poeta); e inoltre XVII 7-8; XXVIII I 3-I 4; XLI 5-6 («O tempestosa, o torbida procella, / che 'n mar sì crudo la mia vita giri!»).

- 9: Lasso!: per un attacco analogo, nella medesima posizione metrica (in apertura di sirma), vd. LVI 9: «Misero! et degno è ben ch'ei frema et arda». A monte, il Petrarca di Rvf 2 16, 9.

- I I digiuno: nesso palese con la sestina precedente (LXI VV. I 8 e 2 I), dove domina l'area metaforica del cibo (vd. qui sotto);

- I 4 esche: cfr. la sestina LXI, in cui, come detto, esca è parola-rima (e al v. 3 sta per la gloria mondana), così come cibo (vd. al v. 5 miglior cibo: $\mathrm{i}$ beni celesti); ${ }^{62}$ inoltre al v. 38 povera esca è il il cibo povero che simboleggia la vita semplice e libera da ambizioni o desideri smodati, contro le esche mortali di LXII I4, i nutrimenti terrestri che non danno pace ma guerra, non vita ma morte ${ }^{63}$ (è la contrapposizione evangelica fra il pane materiale e il pane di vita [Cristo], il solo che salvi l'anima). Si leggano i vv. 19-22 della sestina LXI: «Fallace mondo, che d'amaro cibo [vd. amaro mondo a LXII 6-7] / sì dolce mensa ingombri! Or di quella esca / foss'io digiun, ch'anchor mi grava, e 'n guerra [vd. a LXIII I I digiuno, e mi gravaro al v. 7] / tenne l'alma co' i sensi ha già tanti annil». Nonché, prima, I 7- I 8: «così l'anima purgo, e cangio guerra / con pace, e con digiun soverchio cibo». Da ricordare poi il sonetto XI, dove il Casa definisce la donna amata «cibo e sostegno mio» (v. Io), e conclude: «Né fia giamai, quando 'l cor lasso freme / nel suo digiun, ch'i' mi procuri altra esca» (I 2-I 3). Amore è unico cibo del poeta anche a XLIV 6 e a XLVII 32 (dove egli ricorda che in gioventù era attratto dalla bellezza femminile come l'uccello che scende dall'alto e vola al suo cibo; l'esca dell'amore ritorna ivi anche al v. 6I); mentre nel madrigale extravagante 72 compaiono il cibo soave e la sinonimica esca di Amor, che fanno soffrire più del digiuno (v. 7: «che fia dunque 'l digiun se 'l cibo è guai / [...]?»).

Un consuntivo e un bilancio, dunque, quelli di Già lessi, ed or conosco in me, ma racchiusi nel breve giro del sonetto, della forma cioè che il Casa sentiva come la più congeniale al suo amore per la brevitas e per la sintesi epigrammatica (fra le ottante sue liriche a noi note si contano non per nulla solo cinque testi "lunghi", ossia quattro canzoni e una sestina); caratteristiche, queste, esaltate al sommo grado dalla peculiare ars rhetorica dell'autore, il cui virtuosismo - come scrive il Garigliano - fa brillare anche nella «picciolezza» del sonetto (che «è, per dir cosi, un'ombra di poesia») «la grandezza dell'artificio 
de' gran corpi di poesia). ${ }^{64}$ Accanto agli elementi più evidenti e più noti (soprattutto gli enjambements e gli iperbati, cui il poeta ricorre quasi a ogni verso)," mette conto qui evidenziare soprattutto la ricerca dell'asimmetria, o meglio la stridente coesistenza di spinte simmetriche e asimmetriche, poiché alla palese e già sottolineata bipartizione del testo, rafforzata dalla presenza di ricercati parallelismi, di studiate antitesi e di fittissimi richiami lessicali tra fronte e sirma, ${ }^{66}$ si contrappone una costruzione complessiva del sonetto che ne scardina l'impianto tradizionale. Infatti, se, come detto, i miti di Glauco e di Esaco si dividono con assoluta regolarità le due parti del componimento ( $v v$. I-8 e 9-I4), l'identico spazio - quattro versi - concesso ai due personaggi entra tuttavia in urto con la struttura metrica, e pertanto l'evocazione della figura mitologica e la sua successiva interpretazione allegorica, che nella fronte si spartiscono le due quartine, nella sirma si distribuiscono invece in modo asimmetrico, occupando rispettivamente quattro e due versi ( $v$. 9-I2 e I3-I4). Ne scaturisce un ardito enjambement interstrofico che fa debordare la prima terzina nel verso iniziale della seconda, ${ }^{67} e$ conferisce alla lirica una struttura logico-sintattica non corrispondente a quella metrica, con un andamento che potenzia l'efficacia sentenziosa della chiusa, isolando a mo' di epigramma gli ultimi due versi $e$ facendone la cifra di un'intera esistenza ("si'l core anch'io, che per sé leve fôra, / gravato bo di terrene esche mortali»). ${ }^{68}$

Tale discrasia fra il livello metrico e quello logico-sintattico, come si sa e come già videro gli esegeti cinquecenteschi (a partire dal Tasso), è uno dei tratti distintivi del sonetto casiano, ${ }^{69}$ e una delle componenti che conferiscono alla sua poesia quella speciale "grandezza» fatta di sapienti chiaroscuri, disarticolazioni sintattiche, torsioni ritmiche, sospensioni del senso e fratture strutturali. ${ }^{70}$ Ora, si suole ripetere che questo duplice e contraddittorio movimento (da un lato verso la fedeltà alle forme chiuse tradizionali, dall'altro verso la disgregazione dall'interno delle loro strutture) è sintomo ed espressione dell'inquietudine interiore del poeta, che si tradurrebbe in un'arte "manieristica" e tormentata; tuttavia, sembra più appropriato ricondurre il fenomeno a una matrice non tanto psicologica o addirittura storico-sociologica, ${ }^{7 \mathrm{I}}$ quanto essenzialmente letteraria. In effetti, l'intenzione di un raffinato classicista qual era il Casa sembra chiaramente quella di rileggere Petrarca alla luce della grande lirica greco-latina, e in particolar modo di Pindaro e di Orazio, ai quali l'esegesi umanistica lo accostava fin da Cristoforo 
Landino, ${ }^{72}$ e che ampiamente ricorrono agli stessi strumenti prediletti dal Monsignore, perseguendo uno stile arduo e artificioso fatto di inversioni, ellissi, brachilogie, asimmetrie e, non di rado, sfasature fra il piano logico e quello metrico. ${ }^{73}$ Sicché, anche nel Casa, l'impiego di simili accorgimenti metrico-retorici si configura non come movimento verso la forma aperta dettato da insofferenza nei confronti delle forme chiuse, bensi, al contrario, come aspirazione a uno stile ancora più complesso, duro e ricercato, lontano da ogni fluidità discorsiva e incline piuttosto alla gravitas e alla sublimitas; cosa di cui ben si avvidero $i$ lettori coevi, che ricondussero senza esitazioni la poetica casiana alle categorie dell'asprezza, della difficoltà e dell'artificio. ${ }^{74}$

Asprezza, difficoltà e artificio che non dovevano apparire al Casa come una negazione, ma piuttosto come uno sviluppo della lezione dei Fragmenta, nel solco di quell'alta tradizione classica cui anche Petrarca veniva tradizionalmente ascritto. ${ }^{75}$ A riprova, si può osservare, in conclusione, come nel caso del sonetto LXII (ma anche in altri), l'ineludibile esempio petrarchesco fornisse al Casa suggestioni anche di questa natura, se è vero che nella canzone delle metamorfosi $i$ cinque miti risultano asimmetricamente suddivisi in otto stanze, e che, mentre gli ultimi due si esauriscono in una sola stanza (le stanze, rispettivamente, 6 e 8), i primi tre vengono distribuiti - con effetto di enjambement logico - a cavallo tra due stanze consecutive (nell'ordine, le stanze 2-3, 3-4 e 4-5). Il Casa, insomma, si rivela petrarchista anche quando da Petrarca sembra a noi più decisamente allontanarsi per adottare soluzioni espressive la cui "modernità" tendiamo talora a sopravvalutare, ${ }^{76}$ condizionati come siamo da una visione eccessivamente "monolitica" $e$ "levigata" dei Fragmenta; a conferma ulteriore del fatto che, come ha scritto argutamente Silvia Longhi, "le vie del petrarchismo sono davvero infinite».

Francesco Bausi 
Abbreviazioni adottate nelle note: Carrai $=$ Introduzione, Nota al testo e commento a Giovanni Della Casa, Rime, a cura di S. Carrai, Torino, Einaudi, 2003 (edizione donde sempre si ricavano, talora con alcune modifiche nell'interpunzione, le citazioni delle liriche casiane); Garigliano = Pompeo Garigliano, Lezione nell'Accademia degli Umoristi di Roma sopra il sonetto LVII di Monsignor Giovanni Della Casa "Già lessi, ed or conosco in me, siccome», in Giovanni Della Casa, Opere, Napoli, s.t., I733, III, pp. 47-56; Quattromani = Sertorio Quattromani, Sposizioni delle rime di monsignor Della Casa, ivi, II, passim; Scarpa, Schede = E. Scarpa, Schede per le "Rime" di Giovanni della Casa, Verona, Fiorini, 2003; Tanturli = G. Tanturli, Introduzione, commento e Nota al testo a Giovanni Della Casa, Rime, Milano-Parma, Fondazione Pietro Bembo-Ugo Guanda Editore, $200 \mathrm{I}$.

I. Che le rime casiane, nella princeps del i 558 (inclusa nelle Rime et prose pubblicate a Venezia presso Bevilacqua e ora disponibili anche in anastatica: Giovanni Della Casa, Rime et prose. Latina monimenta, a cura di S. Carrai, Roma, Edizioni di Storia e Letteratura, 2006, pp. I-I 83), presentino una struttura "d'autore" e possano dunque definirsi un "canzoniere", è oggi riconosciuto dalla maggioranza degli studiosi, anche se non tutti credono a un ordinamento rigoroso e calibrato in tutte le sue parti, e alcuni sospettano che la scelta e la disposizione dei testi possano risalire almeno in qualche misura all'iniziativa del curatore Erasmo Gemini (vd. in particolare Carrai, pp. XVII-XVIII e xxxII-XxxIII; e dello stesso studioso anche l'Introduzione alla cit. anastatica delle Rime et prose, pp. xxIII-Xxix). Comunque, fermi restando gli estremi costituiti dal sonetto proemiale e da quello conclusivo, è innegabile il chiaro percorso ascensionale che porta dall'uno all'altro, con la svolta "penitenziale" della canzone XLVII, la cui funzione e la cui posizione corrispondono perfettamente a quelle della canzone 264 dei Fragmenta (vd. S. Longhi, Il tutto e le parti nel sistema di un canzoniere (Giovanni Della Casa), «Strumenti critici», I 3 (1979), pp. 265-300: 298-99; si tenga presente che nell'edizione petrarchesca curata dal Bembo [I $50 \mathrm{I}]$ la seconda parte dei Fragmenta si apre con la canzone 264, mentre nelle ristampe del i 5 I4 e I 52 I è inaugurata dal sonetto 267 in morte di Laura: è evidente dunque che Casa guardi all'aldina del I $50 \mathrm{I}$, anche per la scelta di far iniziare la seconda parte delle sue rime con una canzone). Inoltre, come osserva Tanturli, p. xxx, nella raccolta si succedono nuclei «particolarmente compatti e assicurati da studiati richiami», e il canzoniere appare internamente ritmato dagli snodi costituiti dalle forme lunghe (canzoni e sestine): il blocco delle tre canzoni consecutive, chiuso dalla canzone XLVII (blocco che «fa da spartiacque fra la parte che precede, varia, di continuo oscillante fra lamenti, pentimenti ed esaltazioni, quasi tutta amorosa, e la seguente, tutta dedicata a una severa meditazione morale»: ivi, p. i I 5), e la sestina LXI, che precede la serie dei tre sonetti conclusivi (cosicché la seconda sezione risulta aperta da tre canzoni e chiusa da tre sonetti).

2. Glauco ed Esaco sono vere e proprie «figure» dell'autore (Scarpa, Schede, p. I64). Sul son. LXII vd. in generale ivi, pp. I63-69; e G. Izzi, Percorsi di Glauco (in margine a un sonetto di Giovanni Della Casa), in Mito e letteratura. Studi offerti a Aulo Greco, Roma, Bonacci, 1993, pp. 79-86.

3. Ovidio dice che Esaco, prima di innamorarsi, viveva appartato e privo di qualunque ambizione (libro XI, 764-766: «oderat hic urbes nitidaque remotus ab aula / secretos montes et inambitiosa colebat / rura nec Iliacos coetus nisi rarus 
adibat»); per il Casa poteva dunque essere simbolo dell'uomo che nasce puro, vale a dire libero dal peso dei beni terreni e delle passioni, ma che poi si fa irretire dagli appetiti materiali. Esaco, che, divenuto smergo, ama immergersi nel mare, è l'uomo che, pur potendo levarsi in alto, ricade sempre in basso, perché attratto dalla terra; ha infatti le ali «d'amoroso pallor segnate ancora», cioè continua ad amare (vd. la nota ad loc. di Marco Ariani nel suo commento al sonetto, in Antologia della poesia italiana, diretta da C. Segre e C. Ossola, II, Torino, EinaudiGallimard, I997, p. 765) e dunque è contrassegnato dal proverbiale pallore degli amanti di cui parla Ovidio (Ars amandi I, 729, e anche di Esaco si legge nelle Metamorfosi, XI, 793, che «fecit amor maciem»; Quattromani, p. 238, aggiunge un dettaglio realistico: «perciocché il mantello e la pennatura del corvo marino [= smergo] ha del pallido»). Allo stesso modo, Glauco, appena mangiata l'erba miracolosa (l'esca del Casa, ossia i desideri mondani), sente il desiderio di entrare nel mare (Ovidio, Met. XIII, 945-46) e così perde la sembianza umana, diventando un mezzo animale.

4. Per tutti i commentatori danteschi antichi, Glauco è l'uomo che s'indìa; secondo Benvenuto da Imola, ad es., l'erba in virtù della quale Glauco si fa dio è la sacra Scrittura e la sapienza teologica, che permette a Dante di innalzarsi alla contemplazione del paradiso, e sulla sua scia molti altri così intendono, fra cui anche Vellutello (I 544) e Varchi (I 545). In una rima casiana esclusa dal canzoniere $(74,9)$, Glauco è interpretato invece in senso "positivo": vi si dice infatti che il riso della donna dona «l'erba onde Glauco diventò beato» (alludendo alla sua trasformazione in dio e alla sua immortalità). Si tratta di un sonetto in cui l'amore è visto, alla maniera stilnovistica, quale forza positiva e nobilitante, giacché la donna conduce l'uomo al sommo bene (vv. I-8) e il suo riso "produce e dona» sia l'erba che fece beato Glauco, sia il cibo di Elena (cioè il nepente, che rimuoveva dal cuore la malinconia e l'ira). Se è suo - il sonetto è infatti conteso al Casa da Gandolfo Porrino, che nel is 5 I lo stampò fra le sue rime - si comprende perché il Casa lo abbia escluso dal canzoniere.

5. Dante Alighieri, La Commedia secondo l'antica vulgata, a cura di G. Petrocchi, Firenze, Le Lettere, i 994, vol. IV, pp. Io- I I.

6. Lo stesso Ovidio narra però (XIII, 900-I s) che Glauco, dopo essere divenuto un dio marino, cadde vittima dell'amore per Scilla, e dunque al Casa egli poteva apparire non realmente mondato dalle passioni terrene. Si rammenti del resto che la tradizione mitografica (da Fulgenzio a Boccaccio: vd. Giovanni Boccaccio, Genealogie deorum gentilium, X, 9, a cura di V. Zaccaria, Milano, Mondadori, I998, vol. I, p. 985 ) interpretava Scilla come la donna libidinosa, e Glauco - che di lei s'invaghisce non appena la scorge sulla riva del mare - come l'uomo accecato dalla lussuria (vd. in proposito Izzi, Percorsi di Glauco cit., p. 81, che ricorda anche come nell'Elegia di madonna Fiammetta si accenni parimenti all'innamoramento di Glauco per Scilla).

7. Trad. di Giuseppe Lozza, Milano, Mondadori, I990, pp. 81 5-17. Il rinvio a questo luogo platonico già fu proposto da Bartolomeo Arnigio nel i 568 (vd. oltre, nota I 2) e da Sertorio Quattromani. È attestata anche un'altra e più peregrina interpretazione del mito di Glauco, trasmessa dagli scolii antichi ad Apollonio Rodio (Casa possedeva gli Argonautica con commenti: E. Scarpa, La 
biblioteca di Giovanni della Casa, «La bibliofilia», 82 [1980], pp. 247-79, p. 253), ripresa da Poliziano (Commento inedito alle Selve di Stazio, a cura di L. Cesarini Martinelli, Firenze, Sansoni, I978, p. 229) e ricordata anche dal Varchi (Sopra il primo canto del "Paradiso". Lezione quinta, recitata nel I 545 , in Id., Lezioni sul Dante e prose varie, a cura di G. Aiazzi e L. Arbib, Firenze, A spese della società editrice delle storie del Nardi e del Varchi, i84I, pp. 295-317: 316-17), secondo la quale Glauco mangia l'erba e ottiene l'immortalità, ma infine, divenuto vecchissimo e ormai stanco della vita, si getta in mare. Qui però non sembra che Casa pensi a questa interpretazione del mito (dove pure l'atto di gettarsi in mare è visto negativamente, come un tentativo di suicidio causato dal tedio per la vita, e dove quest'ultimo scaturisce dalla perversa sete di immortalità, un po' come nel mito celebre della Sibilla cumana). Né il Casa segue quanti (ad es. Pausania e la Suda, che pure possedeva: Scarpa, La biblioteca cit., pp. 260 e 262) attribuivano a Glauco la facoltà di «futura praedicere» (come ricorda GARIGLIANO, p. 48).

8. Pompeo Garigliano, Lettioni lette nell'Academia de gli Humoristi di Roma sopra alcuni sonetti di Monsig. della Casa, Napoli, per Gio. Domenico Roncagliolo, r6 6 6. Le lezioni sul Casa, pronunciate nel I6 I 5 , concernono i sonetti I (pp. 7-50), LXII (pp. 5 I-77), LIV (pp. 8 I-105), XLIX (pp. I09-33), LVII (pp. I 36-1 56). Per comodità, in queste pagine, la lezione sul son. LXII sarà citata dall'edizione del 1733 (= GARIGLIANO). Il Garigliano, nato a Capua in data ignota e morto a Roma intorno al I630, fu uomo di Chiesa e professore di filosofia al Collegio Romano; sul Casa compose sette lezioni su altrettanti sonetti (alle cinque ora ricordate devono infatti aggiungersi altre due lezioni, sui sonetti II e LII, recitate presso l'Accademia degli Oziosi di Napoli e parimenti stampate in quella stessa città dal Roncagliolo nel I6 I6). Le lezioni del Garigliano - che nel I6 I 4 pubblicò a Napoli due commentari latini al Fedone e all'Epinomide di Platone - sono dense di cultura filosofica, ma non prive neppure di buone analisi metrico-retoriche (a lui si deve non per nulla anche il Pentimerone, un trattato di poetica e di critica letteraria che prende spunto dalla produzione lirica del suo conterraneo Giovan Battista Attendolo); esse presentano talora forti analogie, anche letterali, con le glosse di Sertorio Quattromani (I 54I-I603), che precedono però quelle del Garigliano (anche se entrambe furono stampate nel i6ı6; le Sposizioni del Quattromani apparvero a Napoli presso Lazzaro Scoriggio) e che furono dunque da lui tacitamente utilizzate (vd. qui ad es. le note 23 e 65 ). Sul Garigliano vd. A. Cardillo, Introduzione all'ed. da lui curata del Pentimerone, Napoli, Edizioni Scientifiche Italiane, 2002, pp. 5-36.

9. Garigliano, p. 52; ivi, a p. 48, si afferma che «il pensiero del poeta è di dimostrare l'anima sua essere aggravata dalle cose mortali, e che non può sollevarsi a Dio, e alla contemplazion sua».

ıo. Trad. di M. L. Gatti Perer, Plotino e la metafisica della contemplazione, introduzione di G. Reale, Milano, Vita e Pensiero, I 996 ${ }^{2}$, p. I 80. Gli studiosi di Plotino osservano che le tre categorie di uomini corrispondono ad altrettante scuole filosofiche antiche: nell'ordine, gli Epicurei, gli Stoici (che mettono al primo posto l'onestà e le regole razionali di scelta tra le azioni) e i Platonici (che si innalzano alle cose spirituali): cfr. ibid., e in generale, su questo passo plotiniano, pp. I79-8 I.

I I. Glauco, per lui, è l'anima non composta, originaria, cui si legano e si sovrappongono poi - quando essa cade sulla terra e nel corpo - elementi, aggiunte e 
scorie ad essa estranee, che per giungere all'Uno l'uomo deve eliminare, purificandosi e tornando alla semplicità originaria; cfr. T. A. Szlezák, Platone e Aristotele nella dottrina del Nous di Plotino, trad. it. Milano, Vita e Pensiero, 1997, pp. 232, 235-36, 248, 262, 273. In Plotino ricorre anche l'analoga immagine della statua d'oro coperta di ruggine: Enneadi, IV, 7, , $о$.

I 2. Vd. anche Scarpa, Schede, p. I66: «anche prima delle attente chiose del Quattromani (1616), i lettori del Casa erano in grado di cogliere le allusioni platoniche del sonetto». La studiosa ricorda in particolare Bartolomeo Arnigio (il quale, membro dell'Accademia degli Occulti di Brescia, già nel i 568 riconduce i versi del Casa al decimo libro della Repubblica platonica: Rime degli Accademici Occulti con le loro imprese et discorsi, Brescia, Vincenzo di Sabbio) e il Tasso (per il quale vd. qui più avanti, a testo). L. Baldacci, Il petrarchismo italiano nel Cinquecento, nuova ed. accresciuta, Padova, Liviana, I974, p. 245, sostiene che una matrice platonica è visibile anche nel sonetto LXIV, dove il tema della seconda quartina «è come un risvegliarsi alla luce in senso platonico, come platonica era stata l'interpretazione allegorica del mito di Glauco», e scrive: "Come non pensare al mito della caverna a proposito di "questa luce / chiara, che 'l mondo agli occhi nostri scopre"?».

I 3. Scarpa, La biblioteca cit., passim. Il Casa possedeva molte opere di filosofia greca: Aristotele (con vari commenti antichi e moderni), Alessandro di Afrodisia, Giovanni Filopono, Platone, i neoplatonici (Ammonio, Simplicio, Porfirio, Proclo), Temistio, Teone di Alessandria.

i 4. Torquato Tasso, I dialoghi, a cura di C. Guasti, vol. I, Firenze, Le Monnier, I 858, pp. i 9-68 (secondo il testo della stampa Delle rime et prose del sig. Torquato Tasso, Venezia, Vasalini, I 583 ); il passo qui cit. a testo si trova a p. 55, l'analisi del sonetto alle pp. 55-63. L'ed. critica curata da Ezio Raimondi (Torquato Tasso, Dialoghi, Firenze, Sansoni, i 958 , vol. I, pp. I 57-245) accoglie solo la terza redazione (risalente al i 586 e intitolata Il Nifo overo del piacere), con le «varianti intermedie d'autore attestate da E e F (redazione di $\beta$ )», dove E = Modena, Bibl. Estense, ms. $\alpha . V .6 .8$, e F $=$ Ferrara, Bibl. Comunale, ms. II.357. Tra queste varianti si trova un passo, poi cassato, che contiene una breve menzione di Glauco (pp. 23 I-32, apparato), donde si ricava che Tasso aderisce già all'interpretazione dantesca del mito: «diventeremo quasi divini, come fe' Glauco nel gustar de l'erba» (p. 232, variante di $\beta$ ). E sopra, p. 23I, variante di E: «l'appetito ragionevole, essendo tirato a basso dal concupiscibile, è quasi sforzato; ma s'egli è rapito da la somma parte de l'intelletto, diviene quasi divino: laonde sentiamo qual si fe' Glauco nel gustar de l'erba [poi sostituito con: divegnamo simiglianti a l'intelligenze]». Vd. anche Torquato Tasso, Discorsi del poema eroico, libro V, in Id., Prose, a cura di E. Mazzali, Milano-Napoli, Ricciardi, I959, pp. $325-26$, dove si parla delle allegorie di Platone, fra cui quella (p. 326) del «Glauco maritimo» (che Tasso non illustra, però).

I 5. Vd. il già ricordato Boccaccio delle Genealogie (qui cit. alla nota 6). L'erba rappresenta il piacere sensuale in quanto pasto degli animali bruti, a loro volta tradizionale emblema degli uomini lussuriosi (si pensi alle metamorfosi di Circe, evocate a questo proposito dal Nifo nella prima redazione del dialogo tassiano: ed. Guasti cit., p. 56). 
I6. Vd. il luogo dei Trionfi qui sotto cit. Quanto alla simbologia del mare, si può ricordare - oltre al passo platonico citato in apertura - anche Agostino, De civitate Dei, XVIII, 23, che spiega come il simbolo del pesce si addica a Cristo giacché solo Lui ha potuto mantenersi vivo (cioè senza peccato) pur immergendosi nella profondità delle acque marine (cioè in questa condizione mortale).

17. In un'edizione delle sue rime da lui stesso chiosate (Brescia, Marchetti, I 592, pp. 66-67). Si badi che il sonetto è scritto «In occasione che la sua donna gli donò un'insalata. Dice rinnovarsi in lui il prodigio dell'erba mangiata da Glauco, la quale il deificò» (così in TorQuato TAsso, L'Aminta e rime scelte, a cura di A. Fabroni e G. Gherardini, Milano, Dalla Società tipografica de' classici italiani, I 824, p. 175 ; invece, nell'ed. delle Poesie curata da F. Flora, Milano-Napoli, Ricciardi, I952, pp. 92-93, donde si cita, la rubrica recita: «Loda l'erba mandatagli in dono e coltivata da la sua donna, facendone comparazione con quella per la quale Glauco si trasmutò»).

I 8. Ma anche Ovidio, Met., XI, 753 definisce lo smergo «spatiosum in guttura».

I9. Vd. infatti il v. 6 «puro anch'io scesi», commentato in chiave platonica da Garigliano, p. 50: «per io non intende il composto di anima e corpo, ma l'anima sola»; e così egli glossa puro: «imperocché, al dir di Platone, l'anime nostre, innanzi che si facessero sensuali ne' corpi, erano pure e belle, cioè di natura intelligibile, ma scese ne' corpi si fanno impure, cioè sottoposte a' sensi» (ibid.). In effetti, il Casa legge Glauco come exemplum negativo perché Glauco "scende" dalla terra nel mare, compie cioè un movimento verso il basso (cfr. anche Ovidio, Met., XIII, 948: «corpusque sub aequora mersi»).

20. Fedro, 246c-256e. Vd. in particolare 246c: «quando [l'anima] è perfetta e fornita di ali vola in alto e ha cura dell'intero mondo; ma nel caso che abbia perso le ali, è spinta fino a che si impadronisca di qualcosa di duro, dove, prendendo dimora, impossessatasi di un corpo terrestre, che sembra muoversi da sé in virtù della potenza di lei, è chiamato vivente nella sua interezza, anima e corpo insieme, e acquista la denominazione di mortale»; 246d-e: «La potenza dell’ala è per natura di condurre in alto ciò che è pesante, volando lassù dove risiede la stirpe degli dei. E in qualche modo, in misura più grande di tutto ciò che riguarda il corpo, essa ha partecipato del divino, e il divino è bello, saggio, buono e tutto ciò che è simile a questo. Di queste cose si nutre e si accresce al massimo grado la parte alata dell'anima, mentre con il turpe e il cattivo, e con cose che le sono contrarie, deperisce e muore»; 248b-c: «Il motivo da cui nasce il grande zelo di vedere dove sia la pianura della verità, è che il pascolo adeguato alla parte migliore dell'anima si dà il caso che sia proveniente da quella prateria là, e che la natura dell'ala, con cui l'anima si rende leggera, si nutre di questo. Tale è la legge di Adrastea, che qualunque anima, divenuta seguace di un dio, abbia scorto qualcosa della realtà vera, fino all'orbita successiva sia sana e salva, e, qualora abbia sempre la capacità di fare ciò, sia incolume per sempre; qualora invece, non essendo riuscita a farsi guidare, non avvia visto, e, colpita da qualche accidente, riempita di oblio e di cattiveria, sia divenuta pesante, e, una volta appesantita, perda le penne e cada verso la terra, allora [...]»; $249 \mathrm{c}$-d: «giustamente mette le ali solo l'intelligenza del filosofo; perché sempre, secondo la sua capacità, con la memoria è rivolto a quelle cose che rendono divino il dio, in quanto si rivolge ad esse. [...] Ponendosi 
al di fuori delle premure umane e divenuto prossimo al divino [...]» (trad. di F. De Luise, in Platone, Fedro, Bologna, Zanichelli, i997, pp. Iо9-14). E vd. anche Boezıo, De cons. phil., IV, m. I, I-4: «Sunt etenim pennae volucres mihi / Quae celsa conscendant poli; / Quas sibi cum velox mens induit, / Terras perosa despicit».

2 I. Tanturli, p. 192 (e anche pp. Xurv-XLVI) definisce quelle di Glauco ed Esaco «metamorfosi degradanti, allusive al proprio regresso», e suggerisce il confronto per antitesi con la trasformazione di se stesso in cigno descritta da Orazio nell'ultima ode del II libro (20, 9-I 2), che allude invece all'immortalità conseguita grazie alla poesia, mentre qui l'aspirazione all'immortalità è causa della metamorfosi degradante di Glauco. Lo stesso Tanturli (p. 192) parla di «intenzione parodica e polemica sul vero sbocco della ricerca di gloria, anche della gloria poetica», rispetto all'ottimismo del modello oraziano, con conseguente denuncia della vanità della gloria poetica - tema forte dell'ultima parte del canzoniere del Casa e ripudio del tradizionale umanesimo letterario di matrice petrarchesca.

22. Garigliano, pp. 50-5 I, a parere del quale le tempeste del v. 7 sono «gli affetti dell'appetito sensuale, i quali con tanta veemenza l'anima muovono, che la rendono fluttuante» e la aggravano (e cita il Fedone, 8Ic-d; ma vd. in generale per questo tema - il corpo che appesantisce e contamina la purezza dell'anima - 8od e sgg.).

23. Ariani (commento cit., p. 765) rinvia a Orazio, Carm. III, 29, 63, ma questa sineddoche è diffusa in latino: vd. ad es. Orazio, Carm., II, i6, 2; Persio, Sat., V, i42; Valerio Flacco, Argon., I, 56r. Garigliano, p. 49, afferma che il Casa nomina l'Egeo perché «è il più tempestoso degli altri [mari]» («non solamente chiama mare la nostra vita, ma sceglie un mare particolare, il più tempestoso di tutti gli altri»: Quatromani, p. 238). La Scarpa, Schede, pp. 163-64, collega il sonetto al soggiorno del Casa a Nervesa, scrivendo che «il complesso percorso mnemonico suggerito dalle favole ovidiane di Esaco e di Glauco [...] riporta il Casa da Nervesa [...] alle acque della laguna, a Venezia, nella prediletta Murano, e suggerisce immagini marine».

24. Passo riferito anche da Petrarca nel primo libro del Secretum (a cura di E. Fenzi, Milano, Mursia, I992, p. I 36), dove pure compare, in bocca ad Agostino, il tema dell'anima umana che «ex contagio corporis» degenera «a primeva nobilitate sua», in conseguenza degli elementi estranei («species innumere et imagines rerum visibilium») che attraverso i sensi penetrano al suo interno e la «pregravant atque confundunt».

25. Vd. ancora Giovanni Boccaccio, Genealogie, V, i 2, ed. cit., I, pp. 540-42, che (sulla scorta di Bernardo Silvestre, Commento all'Eneide, VI, i i 9 , ed. a cura di B. Basile, Roma Carocci, 2008, pp. I 38-40) spiega come Aristeo debba identificarsi, a norma di etimologia, con la virtù, ed Euridice con la «naturalis concupiscentia» dell'uomo, che trova la morte inseguendo i beni terreni e fuggendo la virtù. Osservo che lo stesso Boccaccio, a proposito del mito di Esaco, riferisce interpretazioni allegoriche di natura esclusivamente evemeristica, e non filosofico-morale (Genealogie, VI, 32, ed. cit., I, p. 668). 
26. Carrai, nel suo commento al sonetto XXXVI (Giovanni Della Casa, Rime, ed. Carrai, cit., p. I09), rinvia anche ad analoghi sonetti del Dolce e del Varchi, e a Fur. XI, 70, dove Olimpia è detta più bella di Venere, vincitrice delle tre dee nel giudizio di Paride; si può aggiungere anche JACOPO NARDI, Canzona sopra il carro delle tre dee (in Trionfi e canti carnascialeschi del Rinascimento, a cura di R. Bruscagli, Roma, Salerno Editrice, I986, I, pp. 76-78), dove le tre dee concedono i loro doni alla donna celebrata, probabilmente Madeleine de la Tour d'Auvergne, che andava nel i 5 I 8 in sposa a Lorenzo de' Medici il Giovane. Tanturli, sulla scorta del Quattromani, rinvia inoltre a Ovidio, Her., XVI, I39-40 (lettera di Paride a Elena), che allude al celebre giudizio e afferma che, se Elena vi avesse partecipato, la vittoria di Venere sarebbe stata in dubbio: «Si tu venisses pariter certamen in illud, / in dubio Veneris palma futura fuit» (ma vd. del Casa anche il sonetto XXIX, ıо-I I, dove egli proclama che la bellezza della donna da lui amata pareggia quella delle dee del giudizio di Paride). Lo stesso Casa, in una sua lettera al Gualteruzzi del i 8 dicembre I546, fa riferimento a una primitiva stesura del sonetto XXXVI, in cui le donne erano quattro, aggiungendovisi, ai vv. 3-4, Callisto (ninfa amata da Zeus), che poi fu soppressa per ridurre il peso della componente mitologica: «credo che quelle tante favole siano inculcate et levino la vaghezza di quei versi, facendoli satievoli, et però pensava di levarne Calisto che è nel terzo et nel quarto verso» (cit. da Carrai, p. i ıo).

27. Il rinvio a Rvf 23 fu già proposto da Garigliano, p. 49.

28. A. Sole, Cognizione del reale e letteratura in Giovanni Della Casa, Roma, Bulzoni, I98 I, p. 53, scrive che i miti di Glauco ed Esaco nel son. LXII sono «emblemi, carichi di religioso pathos, del dramma umano del riscatto dal terreno», e a p. 54 ne parla come di «oggetti-simbolo»; altrove (L" "imitatio Bembi" nelle «Rime» di Giovanni Della Casa, "Giornale storico della letteratura italiana», I 23 [2006], pp. 48 I-539: 534-35) il medesimo Sole scrive che le immagini e i simboli di questi miti «sono già pregni del significato morale che il Casa chiosa in riferimento a se stesso».

29. Qui e oltre cito dall'ed. dei Fragmenta curata da R. Bettarini, Torino, Einaudi, 2005,2 voll.

30. Anche in $R v f 23$, dunque, Petrarca sperimenta finalmente su se stesso quelle metamorfosi che in precedenza aveva considerato semplici invenzioni letterarie, senza sospettare che potessero avere un contenuto eticamente vero e potessero quindi costituire exempla utili alla vita (nella fattispecie, a guardarsi dall'amore, che fa soffrire l'uomo e lo priva della ragione, riducendolo ad animale bruto o ad essere inanimato). Donde l'interpretazione esatta del v. I 20: «parlo cose manifeste et conte» non si riferisce infatti al racconto ovidiano (come ritiene Marco Santagata: Francesco Petrarca, Canzoniere, a cura di M. S., Milano, Mondadori, I 996, p. i 16), ma a sé stesso (la cui trasformazione in una fonte di lacrime è sotto gli occhi di tutti, è reale, è appunto cosa manifesta e conta), perché è lui l'nom vero del v. precedente (I I 9); Petrarca sta cioè dicendo che, prima di aver vissuto una simile esperienza, non aveva creduto che da un uomo vero - e non da un personaggio immaginario come Biblide - potesse scaturire una fonte.

3i. Petrarca, Secretum cit., p. 242. È significativo che anche qui si tratti di versi ovidiani, come nel sonetto LXII del Casa, e come ovidiana è la lettura cui 
Petrarca fa riferimento in $R v f 56$, I 2 a proposito del detto di Solone: perché Ovidio era autore notissimo, e in particolare le Metamorfosi erano testo di lettura scolastica. Da qui l'antitesi «già lessi, e or conosco»: il Casa - come Petrarca conosceva benissimo e da molto tempo quei passi, ma solo ora ne comprende il senso profondo e ne ricava un insegnamento morale, mentre in passato la sua era stata una fruizione puramente letteraria.

32. Petrarca, Secretum cit., p. 220.

33. «A. Lectio autem ista quid profuit? Ex multis enim, que legisti, quantum est quod inheserit animo, quod radices egerit, quod fructum proferat tempestivum?»; «F. Singula hec haud negligenter legisse me noveris. [si riferisce agli scritti di Seneca e di Cicerone] $A$. Quid ergo? nichil ne profuerunt?. F. Imo vero inter legendo plurimum; libro autem e manibus elapso assensio simul omnis intercidit. $A$. Comunis legentium mos est, ex quo monstrum illud execrabile, literatorum passim flagitiosissimos errare greges et de arte vivendi, multa licet in scolis disputentur, in actum pauca converti» (pp. I44 e 192).

34. Vd. Petrarca, Secretum cit., p. 230: «A. Qualiter autem lecta intellectaque in salutem tuam vertenda sint, admonuisse forsitan non erit alienum»; e p. 252 , dove ad Agostino, che gli aveva ricordato il passo di Seneca (Nat. quaest., I, 17, 4) relativo allo specchio e alla sua utilità morale per migliorare se stessi, Francesco dice di conoscerlo bene, e il medesimo Agostino replica: «Quid vel legisse vel meminisse profuit? Excusabilius erat ignorantie clipeum posse pretendere».

35. Petrarca, Secretum cit., pp. 258-60.

36. Pétrarque, Les remèdes aux deux fortunes - De remediis utriusque fortune, texte établi et traduit par Ch. Carraud, Grenoble, Jerôme Millon, 2002, I, p. 226.

37. Pétrarque, Les remèdes cit., I, p. 596. Petrarca allude a due aneddoti narrati da Vitruvio, De architectura, VI, praef. I-2. Aristippo, gettato in seguito a un naufragio sulle spiagge di Rodi, si diresse in città e vi tenne una lezione di filosofia, ricevendone doni in abbondanza; cosicché, quando i suoi compagni, mentre si apprestavano a tornare in patria, gli chiesero che cosa volesse mandare a dire a casa sua, rispose che dicessero loro di fornire ai figli possedimenti e viatichi tali che potessero scampare a un naufragio insieme con loro. Teofrasto, analogamente, esortava a istruirsi piuttosto che a confidare nel denaro, affermando che solo il dotto non è estraneo nel paese altrui, né sarà privo di amici se un giorno perderà familiari e parenti, e potrà guardare senza timore ai casi avversi della fortuna; mentre chi ritiene di essere più garantito dalle ricchezze che dalla dottrina, camminando su una via sdrucciolevole, conduce una vita rischiosa ed instabile. I due aneddoti sono riferiti da Petrarca per esteso in questo stesso capitolo del De remediis, rispettivamente ai parr. i 2 e 16 (pp. 595 e 596).

38. Scarpa, Schede, pp. i65-66: «All'antica lettura di svago, relegata a tempi remoti ("Già lessi...»), si contrappone con immediato parallelismo l'odierna presa di coscienza» («or conosco...»). Qui si innesta, fra l'altro, la polemica del Casa contro la letteratura umanistica del secondo Quattrocento, accusata (nella Petri Bembi vita, ed. a cura di A. Sole, Torino, Fògola, I997, pp. 46-47) di un gusto superficiale per 
la vacua erudizione mitologica degli autori più peregrini. Vd. al riguardo il mio $I$ carmi latini di Giovanni Della Casa e la poesia umanistica fra Quattro e Cinquecento, in Giovanni Della Casa, Atti del convegno di Firenze, a cura di S. Carrai, Roma, Edizioni di Storia e Letteratura, 2007, pp. 233-58: 245-46 e 25 I.

39. Tanturli, p. XLVII (e vd. qui anche oltre, nota 48). Di Michelangelo cfr. soprattutto il sonetto is I e il madrigale is 2 , incentrati sul motivo platonico della necessaria eliminazione del superchio; nel madrigale, in particolare, si propone un'esplicita analogia fra la tecnica del levare propria dello scultore e la condizione dell'uomo, che per salvare la propria anima deve liberarsi del «superchio della propria carne», da quella «inculta sua cruda e dura scorza» (simile dunque alla pelle di Glauco disceso nel mare, coperta di spume e conche). Ma l'uomo - se non lo soccorre la donna, avviandolo alla perfezione spirituale - non ha né il voler né la forza di sollevarsi verso l'alto (levarne, v. io) dalle streme parti (il corpo, la terra) in cui giace. Vd. Michelangelo, Rime, a cura di M. Residori, Milano, Mondadori, I998, p. 265, col relativo commento del curatore.

40. Dei versi dedicati ad Esaco (Tr. Cup., II, I60-62: «lungo costor pensoso Esaco stare / cercando Hesperia, or sovra un sasso assiso, / ed or sotto acqua, ed or alto volare») forse si percepisce qualche eco nella sirma del sonetto LXII. La Scarpa, Schede, pp. 164-65, ricorda anche altri due testi moderni: l'Hypnerotomachia Poliphili (uscita presso Aldo nel I499), dove nella stessa pagina (ed. a cura di M. Ariani e M. Gabriele, Milano, Adelphi, I998, I, p. 280) sono accostati Glauco ed Esaco (la metamorfosi di Glauco è lì interpretata in termini negativi, come metamorfosi mostruosa, tanto che Glauco è incluso fra i mostri marini [«pissatili monstri»]), e dove Esaco è l'amante doloroso («il dolente Esaco, cum la voce luctifica, vestitosi di anthracino colore, overo di fusca veste pullato per la sua cara Epiriphe [errore per Esperie] morsicata dal venenoso serpe»; Esaco ricorre anche a p. 333: «le piume anthracine di Esacho», e a p. 381: «la terrifica raucitate de l'urinante Esacho»); e un sonetto di Niccolò Franco, incentrato sul solo Esaco, che ancora va in cerca della donna amata ( $\mathrm{I}-4$ : «Esaco avventuroso, che nel mare / ch'io varco con Amor, mostri al mio viso / che da l'antico stil non t'ha diviso / la morte ch'a te pur ti piacque dare»).

4I. Come scrive nelle sue annotazioni Marco Aurelio Severino (Tarsia i $580-$ Napoli i656, medico e letterato, membro di quell'Accademia degli Oziosi frequentata, come abbiamo detto, anche dal Garigliano), «in somma questo componimento è rapidissimo, e di contratta velocità, quant'ogni altro, né veruno in ciò l'avanza, se non il sezzaio, che prerogative tiene per lo divin soggetto» (in GIOvanni Della Casa, Opere [1733] cit., II, pp. 239-40: 240).

42. Cfr. in proposito A. Sole, Note su "Errai gran tempo» di Giovanni Della Casa (2004), in Id., Studi su Bembo e Della Casa, Caltanissetta, Sciascia, 2006, pp. I 50-68: I 54 e I67, che in particolare riconduce al Secretum il motivo dei due "errori": l'amore e il desiderio di gloria (sia poetica che mondana).

43. Longhi, Il tutto e le parti nel sistema di un canzoniere cit., p. 287, parla per questa canzone di «accentuato aspetto di riflessione strutturale sullo svolgimento dell'opera ancora in corso» e di «recapitolazione, graduata secondo una progressione ascendente, dei tre miti costruiti nel canzoniere», che «si intesse di 
fittissime riprese dai componimenti precedenti»; è infatti «un'antologia dei topoi del canzoniere, che ha lo scopo espresso di rappresentarne, in un concentrato ristretto, l'intero universo segnico». Secondo Tanturli, p. xuI, la canzone deve considerarsi la «svolta del libro in senso morale».

44. Anche se ha certamente ragione Longhi, Il tutto e le parti nel sistema di un canzoniere cit., p. 278 , quando osserva che «dopo la canzone XXXII la tematica amorosa perde terreno, a favore delle altre due tematiche» (la ricerca della gloria poetica e la ricerca degli onori mondani), e che «dell'effuso, protratto, articolato discorso sull'amore persiste, dopo la condanna espressa in XLVII, assai poca cosa» (p. 288), cosicché «il tema quantitativamente più ingombrante nella prima parte del canzoniere è divenuto [...] il più esile e marginale nella seconda» (p. 29I).

45. Così intende Izzi, Percorsi di Glauco cit., p. 80, per il quale «l'esperienza di vita [...] che il Della Casa voleva esprimere nel suo sonetto era più vasta di quella amorosa».

46. Il sonetto LXIV rappresenta Dio che ha tratto la luce dalle tenebre, il bene dal male, il senso dal non-senso, la forma dall'informe: lo ha fatto col macrocosmo al momento della creazione, la fa ogni giorno col microcosmo di ogni uomo, giacché il Casa sperimenta come dai suoi errori e dalle sue pene precedenti egli approdi ora al bene, o meglio come anche quegli errori e quelle pene facessero parte di questo bene, fossero cioè parte di un unico disegno provvidenziale.

47. S. Carrai, Il canzoniere di Giovanni Della Casa dal progetto dell'autore al rimaneggiamento dell' edizione postuma, in Per Cesare Bozzetti. Studi di letteratura e filologia italiana, a cura di S. Albonico et alii, Milano, Fondazione Arnoldo e Alberto Mondadori, 1996, pp. 47 I-98: 492-93, non esclude invece che l'ordinamento del Chigiano possa essere quello d'autore, e che il Gemini sia intervenuto per assegnare alla raccolta una chiusa "religiosa" più conveniente al clima controriformistico. La successione dei due sonetti finali nella princeps costituirebbe dunque una banalizzazione; ma io ritengo piuttosto che sia la nostra sensibilità moderna a farci preferire un Della Casa deciso a chiudere le sue rime su una nota pessimistica e non "confessionale". G. Tanturli, Dai "fragmenta" al libro: il testo di inizio nelle rime del Casa e nella tradizione petrarchesca, in Per Giovanni Della Casa. Ricerche e contributi, a cura di G. Barbarisi e C. Berra, Milano, Cisalpino, I 997, pp. 6I-89, pp. 87-89, pur pronunciandosi, quanto ai due sonetti conclusivi, a favore dell'ordinamento della prima stampa, non esclude che il poeta sia rimasto incerto fino all'ultimo sulla disposizione da adottare.

48. Tanturli, pp. XLVI-XLVII: «la sezione morale, che costituisce l'approdo del libro e della vicenda in esso rappresentata, si configura infatti come progressiva catarsi, dissolvimento di obiettivi ingannevoli anche sotto le più nobili apparenze e proposti dalle più alte elaborazioni culturali, alla ricerca dell' "uom puro e chiaro" (LXII, 2), nascosto e gravato da sovrastrutture e orpelli, che ne soffocano l'aspirazione all'assoluto, secondo i miti bellissimi di Glauco e di Esaco del sonetto LXII. È la stessa cosa, mi pare, del concetto di creazione artistica e di elevamento morale di Michelangelo». Donde, continua Tanturli, l'insistenza sul trarre - e sull'aprire ciò che era chiuso - nell'ultimo sonetto (vv. I I e I 3). 
49. Vd. per questo anche LVI, 5-8: «Et bene il cor, del vaneggiar mio duce, / vie più sfavilla che percossa selce, / sì torbido lo spirto riconduce / a chi sì puro in guardia e chiaro dielce».

50. Si può aggiungere inoltre, al di fuori del canzoniere, il sonetto 75, dove il Casa chiede a Dio che la sua anima - definita platonicamente «il mio miglior» - possa fare ritorno al «nido vero» donde uscì fuori, perché ora finalmente è tornata «nuda e sincera», essendosi squarciato il velo che ottenebrava gli occhi della mente e quelli del corpo, impedendo loro di scorgere il cammino verso il «porto vero».

51. Sole, Cognizione del reale e letteratura cit., p. 53: «L'esempio di Esaco formalmente è una variante retorica in chiave mitologica del solito emblema dell'uccello "in ima valle preso"»,

52. Cfr. C. Scarpati, Il ritorno di Dante. Note su Castiglione, Della Casa, Tasso, in Id., Dire la verità al principe. Ricerche sulla letteratura del Rinascimento, Milano, Vita e Pensiero, I987, pp. I 27-56: 136, che pone l'accento «sulla presenza invasiva nell'ultima parte delle Rime casiane della metafora ornitologica e sul suo sviluppo rigoglioso lungo la via di variazioni molteplici fino a divenire una vera "metafora ossessiva"» (lo studioso ricorda inoltre i «difettivi sillogismi» che «fanno in basso batter l'ali» di Par. XI, I-3, e il Fedro platonico); e Longhi, Il tutto e le parti nel sistema di un canzoniere cit., pp. 29I-92, che definisce quello dell'angello «simbolo chiave» della seconda parte delle rime: «sottratto alla significazione di prigioniero o transfuga che gli era connaturata nella prima parte, esso passa ora invece a rappresentare il poeta contaminato dalla passione dell'ambizione. [...] Mutando la funzione simbolica del motivo, muta la sua esecuzione: è sparita ogni connotazione patetica (presente nei testi amorosi in diminutivi quali angellino, augelletto), ed è cambiato lo scenario del volo, che non si svolge più tra rami (nascosti, verdi), ma, fatto decisivo, in una valle, anzi con sintagma fisso in un'ima valle (XLIX, Iо; LXI, I4), e nel fango e palustre limo che la riempiono. Abbiamo già visto come la valle profonda e paludosa [cfr. XXVI, I: "Mentre fra valli paludose e ime"] sia segno distintivo, fin dal suo apparire, del tema $\mathrm{C}$, polare rispetto al monte di A e di B» (sono $\mathrm{i}$ tre temi delle rime casiane, gli oggetti della sua ricerca: amore, gloria poetica, onori mondani).

53. Analogo motivo è svolto nei sonetti XXVI (dove il poeta rimane «fra valli paludose et ime», mentre il dedicatario Bernardo Cappello sale sciolto verso le «spedite cime») e XXXVII (dove l'anima del Bembo, dopo la morte, «al ciel sen vola»).

54. Importante, a questo riguardo, il modello petrarchesco dei Fragmenta; vd. Rvf 359, 39-42 (parla Laura a Francesco: «Quanto era meglio alzar da terra l'ali, / et le cose mortali / et queste dolci tue fallaci ciance / librar con giusta lance»); $R v{ }_{3} 65$, I-3: «I' vo piangendo i miei passati tempi, / i quai posi in amar cosa mortale, / senza levarmi a volo, abbiend'io l'ale» (e anche $R v f 360,28-30$ e i 37-39, i43). È, questo, tema della parte finale dei Fragmenta, ma anticipato già a Rvf 8I, I 2-I 4 ("Qual gratia, qual amore, o qual destino / mi darà penne in guisa di colomba, / ch'i' mi riposi, et levimi da terra?»), dove pure è introdotta l'antitesi fra le ali (qui, le penne di colomba del salmo 54, 7) e il «fascio antico» del v. I: «Io son sì stanco sotto 'l fascio antico / de le mie colpe et de l'usanza ria» (le some del Casa). 
55. Longhi, Il tutto e le parti nel sistema di un canzoniere cit., pp. 297-98, parla di «lessico lirico aristocraticamente selettivo»; e a p. 297 sottolinea la «azione unificante svolta sia da alcune immagini [...], sia, capillarmente e in maniera diffusa, dalla continuità di un lessico fedele a se stesso dall'inizio alla fine del libro, costituente un sistema coeso, che è passibile di cauti ampliamenti omogenei, ma intollerante di qualsivoglia innesto eterogeneo».

56. Come rileva Tanturli, p. I92.

57. Cfr. Rvf i 35, 59-60, ma solo per la forma: «tutto dentro et di for sento cangiarme, / et ghiaccio farme, così freddo torno»; e qui XXXII, 42-44: «agghiacciarsi sento / et pigro farsi ogni mio senso interno, / com'angue suole in fredda piaggia il verno» (col verbo sentire che è tipico delle descrizioni di metamorfosi: vd. anche XLVI, 62, cit. alla nota seguente).

58. Vd. in particolare 61-62 («Membrando vo che men di lei fugace / donna sentì fermarsis, in riferimento a Dafne), dove membrando vo richiama il soviemmi di LXII, 9 .

59. Tanturli, p. I94: «dopo il senso d'angoscia della prima quartina, anzi dopo il travaglio ansioso di tutto il libro, rivolto ossessivamente su di sé, dissolte le atre nubi delle false illusioni e aspirazioni, è l'aprirsi improvviso di un diverso e vasto orizzonte, della dimensione della lode».

6o. $\mathrm{Al}$ v. 2 del son. LXII è degna di nota la variante d'autore dei mss. $\mathrm{C}^{2}-\mathrm{Mp}$ (= Biblioteca Apostolica Vaticana, Chigi L IV I 33, e Montpellier, Bibliothèque Interuniversitaire, Section Médecine, ms. 354) «franco e chiaro» (cfr. GiovanNi Della Casa, Le rime, a cura di R. Fedi, Roma, Salerno Editrice, I978, I, p. 78). Anche franco è voce ricorrente nel canzoniere del Casa (vd. VI, I; XXXI, I 2; XXXII, 63), ma nell'accezione di 'coraggioso' oppure di 'libero'; cosicché puro appare qui più congruente al contesto.

6. A. Afribo, Commentare la poesia del Cinquecento, in «Per leggere», 3 (2003), pp. I4I-63: I 55 e I $6_{3}$, cita inoltre l'incipit di Gaspara Stampa «La mia vita è un mar; l'acqua è 'l mio pianto», e alcuni luoghi paralleli di Bernardo Cappello.

62. Esca e cibo come parole-rima di sestina ricorrono soltanto nel Casa (Longhi, Il tutto e le parti nel sistema di un canzoniere cit., p. 297); sono sinonimi, ma il loro significato muta talora nel corso della sestina LXI. Il termine esca (nel senso metaforico di 'lusinga terrena' e 'tentazione amorosa', ma a volte anche in quello proprio di 'esca dell'amo') è frequente nelle rime casiane: cfr. XXXII, 63 (dove il poeta ricorda quando, uccello ancora implume, fu preso all'esca d'Amore); XL, 4; XLVII, 6I; 72, 6 .

63. Secondo Garigliano, p. 53, le «esche» sono «mortali» in quanto «sottoposte alla corruzione», o perché «cagioni della morte eterna dell'anima», o infine perché «rendon mortale l'anima nostra, mentre vivendo data alle cose sensuali è morta secondo lo spirito e l'intelletto».

64. Garigliano, p. 53: «sebbene questo sonetto, ed ogni altro che qui prenderò a spiegare, è, per dir così, un'ombra di poesia per la picciolezza sua, nondimeno si conoscerà per mezzo suo la grandezza dell'artificio de' gran corpi di poesia». 
65. Come scrive Afribo, Commentare la poesia del Cinquecento cit., p. I45: «ad ogni iperbato (o epifrasi) in Bembo ne corrispondono quattro in Della Casa» (con rinvio anche al Galateo, dove quali esempi di simile artificio - sconsigliato nella lingua parlata e ritenuto conveniente talora solo a quella poetica - si citano il dantesco «imagini di ben seguendo false» [Purg. XXX, I 3 I] e il petrarchesco «del fiorir queste inanzi tempo tempie» [Rvf 210, I4]: ed. a cura di G. Barbarisi, Venezia, Marsilio, i991, p. 93). Anche il Garigliano, pp. 54-55, evidenzia nel sonetto LXII l'uso sapiente e frequente dell'iperbato o «interposizione» («l'iperbato è quando in mezzo della dritta orazione s'interpongono più parole», p. 55 ) ai vv. 6-7 «e'nqueste de l'amaro / mondo tempeste» (ibid.: «mettendo fra l'aggiunto e il nome alcune parole, per far grandezza»; sulla scorta di QuATtromani, p. 238 : «Quando fra l'aggiunto e 'l nome si traspongono alcune parole, fanno grandezza») e I 3-I 4. Lo stesso Garigliano sottolinea inoltre il «polisigma» del v. 3, che egli definisce anche «annominazione». Vd. a questo proposito A. Afribo, «Si compiaceva più della gravità. Note sulla lirica di Giovanni Della Casa, «La parola del testo», 2 (I 998), pp. 309-48, in part. 33 I-48.

66. Vd. ad es. le due interiezioni collocate a specchio ai vv. 8 e 9 (ahi-lasso), rispettivamente al termine della fronte e all'inizio della sirma, dunque nel cuore del sonetto. E inoltre: I e 9 lessi-soviemmi; 2 e 5 mar-Egeo; 2 e 6 puro; 7 e 14 mi gravaro-gravato ho; 2-6 e I 3 puro-leve; 8 e i 3 i sensi e l'alma-'l core; 8 e 14 indegne some-esche mortali; I I e i 2 digiuno-satollo; i I e I 4 cielo-terrene esche; I 3 e I 4 levegravato. Si aggiungano poi le dittologie di 2 puro e chiaro, 4 spume e conche, 8 i sensi e l'alma, i I apre e distende; la paronomasia lessi - lasso (vv. i e 9, in apertura di fronte e di sirma); e le allitterazioni di i conosco ... come, 2 pose ... puro, 3 sue sembianze (e anche miSchiaro), 4 conche ... chiome (con assonanza e paronomasia in sede di rima interna), 8-9 sensi ... some ... soviemmi.

67. G. Stella Galbiati, L'esperienza lirica di Giovanni Della Casa, Urbino, Montefeltro, I 978 (che alle pp. 98-i I 2 ha buone osservazioni sulla metrica del Casa) sottolinea a p. 99 che l'«aggancio logico-sintattico» fra le terzine caratterizza anche il sonetto XXXVI.

68. La grave solennità dell'ultimo verso è accresciuta dagli accenti ribattuti di $6^{\mathrm{a}} \mathrm{e}$ $7^{\mathrm{a}}$ con sinalefe "foscoliana"; QuATtromani, p. 238 , scrive a questo riguardo: «Fa il verso di numero tardo, che pare che non possa fare il suo viaggio». Vd. Stella Galbiati, L'esperienza lirica di Giovanni Della Casa cit., che alle pp. I03-107 elenca gli endecasillabi con accenti di $6^{\mathrm{a}}$ e $7^{\mathrm{a}}$ nelle rime del Casa, fra cui LXII, 4 e I4 (ma si aggiunga anche r r: cioè, si badi bene, sempre in fine di periodo metrico, quartina o terzina). La studiosa osserva poi come questa accentazione sia una delle caratteristiche peculiari dell'endecasillabo casiano e costituisca la forma più consueta con cui nelle Rime si manifesta l'inclinazione, già petrarchesca, ai versi con ictus ripercossi (si tratta di un endecasillabo prediletto dal Tasso, e poi da Parini e Foscolo, in virtù soprattutto della sua clausola di tipo esametrico). Il Casa, invece, non ama il cosidetto endecasillabo dattilico, con accenti cioè di $4^{\mathrm{a}}$ e $7^{\mathrm{a}}$, d'altronde poco usato anche da Petrarca.

69. Come scrive Tanturli, p. xxiv, il Casa si ingegna di «evitare quel che di scontato e di schematico è nella struttura del sonetto», e per questo «la sintassi tende a non combaciare con la struttura metrica». 
70. U. Schulz-Buschhaus, Le «Rime» di Giovanni Della Casa come "lectura Petrarcae", «Studi petrarcheschi», IV (I 987), pp. I43-58, pp. I 54-58, dove anche si sottolinea la struttura frequentemente asimmetrica del sonetto del Casa.

71. Come fa ad es. Sole, Cognizione del reale e letteratura cit., p. 70: «le Rime [...] si connotano per una tensione interna fra la norma (Petrarca-Bembo) e la vita affettivo-intellettiva che cerca di porre la norma in sintonia col mondo esterno (quindi con la propria funzione intellettuale) e con quello della coscienza e del gusto, non più appagato dalla coerenza idealizzante del modello rinascimentale».

72. Cristoforo Landino, Comento sopra la "Comedia», a cura di P. Procaccioli, Roma, Salerno Editrice, 200I, I, pp. 253-54 (Proemio). E vd. inoltre i passi dei commenti petrarcheschi di Giovanni Andrea Gesualdo e Bernardino Daniello citati da Roberto Cardini nell'ed. da lui curata di Cristoforo Landino, Scritti critici e teorici, Roma, Bulzoni, I974, II, pp. 190-92.

73. Schulz-Buschhaus, Le "Rime» di Giovanni Della Casa cit., p. I47, cita Orazio Marta, Parallelo tra Mess. Francesco Petrarca e Mons. Giovanni Della Casa (1616), in Giovanni Della Casa, Opere, Venezia, Pasinello, i 728 , I, p. 396, secondo cui il Casa «a guisa di nuovo Pindaro s'innalza che nulla più, con virtù inestimabile». In effetti, come osserva la studiosa, il Casa porta avanti il progetto già bembiano «di alzare il registro della lirica a quello della poesia epica e della poesia didattica» (p. I48); e ciò, aggiungiamo, in ossequio all'idea "classica" (greco-latina) di "lirica" come genere non meramente "elegiaco" ed esclusivamente "amoroso", ma "alto" e "composito". La stessa idea teorizzata e praticata, nei Sepolcri, da quel grande ammiratore del Casa che fu Ugo Foscolo (cfr. il mio Dall" "epistola" al "carme". Sul genere metrico-letterario dei «Sepolcri», in I «Sepolcri» di Foscolo. La poesia e la fortuna, Atti del Convegno di Studi, Firenze, 28-29 marzo 2008, a cura di A. Bruni e B. Rivalta, Bologna, Clueb, 2010, pp. 97-I 27).

74. Nicola Villani (I63 I) parla per il Casa - poeta che egli non apprezza - di stile «aspro, difficile e artificioso» (cit. da Schulz-Buschhaus, Le «Rime» di Giovanni Della Casa cit., p. I48).

75. Come scrisse G. Parenti, I carmi latini, in Per Giovanni Della Casa cit., p. 2 Io, in tal modo il Casa «ottenne che il volgare di Petrarca e di Bembo, ripensato e ricalcato sul modello oraziano, sembrasse parlare latino». E vd. anche il mio I carmi latini di Giovanni Della Casa e la poesia umanistica fra Quattro e Cinquecento cit., pp. 256-58; oltre a Scarpati, Il ritorno di Dante cit., p. I 38, che per questo aspetto sottolinea l'importanza di un testo ben noto al Casa (e al Tasso, che ripetutamente lo cita proprio nella sua lezione sul sonetto Questa vita mortal) come il De elocutione di Demetrio Falereo.

76. M. Ariani, Petrarchisti e manieristi, in Antologia della poesia italiana, II, cit., pp. 73 I-32, scrive che il manierismo è una «riformulazione radicale» del petrarchismo, «ma in termini, tutto sommato, già previsti dal codice», e che esso consiste nell'«alterare, ma senza romperli, $i$ dispositivi dell'ordo per un lessico meno condizionato dal vocabolario petrarchesco, per dissimmetrie ritmicosintattiche e logico-strofiche insistite, per un metaforismo più spinto e avventuroso». Nell'àmbito di tale "maniera", continua Ariani, il Casa deve considerarsi «l'iniziatore più fedele alla lezione del maestro e più pervicace nel sommuoverne astutamente gli interni equilibri». 